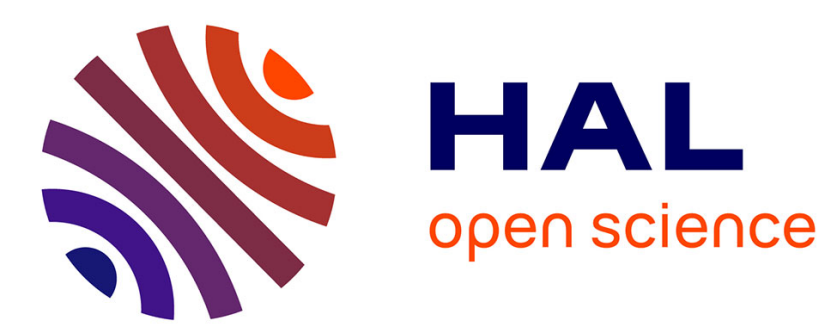

\title{
Discrete spectrum of a model Schrödinger operator on the half-plane with Neumann conditions
}

Virginie Bonnaillie-Noël, Monique Dauge, Nicolas Popoff, Nicolas Raymond

\section{To cite this version:}

Virginie Bonnaillie-Noël, Monique Dauge, Nicolas Popoff, Nicolas Raymond. Discrete spectrum of a model Schrödinger operator on the half-plane with Neumann conditions. Zeitschrift für Angewandte Mathematik und Physik, 2012, 63 (2), pp.203-231. 10.1007/s00033-011-0163-y . hal-00527643v4

\section{HAL Id: hal-00527643 \\ https://hal.science/hal-00527643v4}

Submitted on 18 Jun 2011

HAL is a multi-disciplinary open access archive for the deposit and dissemination of scientific research documents, whether they are published or not. The documents may come from teaching and research institutions in France or abroad, or from public or private research centers.
L'archive ouverte pluridisciplinaire HAL, est destinée au dépôt et à la diffusion de documents scientifiques de niveau recherche, publiés ou non, émanant des établissements d'enseignement et de recherche français ou étrangers, des laboratoires publics ou privés. 


\title{
Discrete spectrum of a model Schrödinger operator on the half-plane with Neumann conditions
}

\author{
Virginie BONNAILLIE-NOËL*, Monique DAUGE ${ }^{\dagger}$ \\ Nicolas POPOFF ${ }^{\dagger}$, Nicolas RAYMOND ${ }^{\dagger}$
}

June 18, 2011

\begin{abstract}
We study the eigenpairs of a model Schrödinger operator with a quadratic potential and Neumann boundary conditions on a half-plane. The potential is degenerate in the sense that it reaches its minimum all along a line which makes the angle $\theta$ with the boundary of the half-plane. We show that the first eigenfunctions satisfy localization properties related to the distance to the minimum line of the potential. We investigate the densification of the eigenvalues below the essential spectrum in the limit $\theta \rightarrow 0$ and we prove a full asymptotic expansion for these eigenvalues and their associated eigenvectors. We conclude the paper by numerical experiments obtained by a finite element method. The numerical results confirm and enlighten the theoretical approach.
\end{abstract}

\section{Introduction and main results}

The aim of this paper is to study the eigenpairs of a Schrödinger operator with a degenerate electric potential of the form $(t \cos \theta-s \sin \theta)^{2}$ on the half-plane $t>0$. This problem is motivated by the analysis of the third critical field $H_{C_{3}}$ in the theory of superconductivity (see for instance [15]). More precisely, the linearization of the Ginzburg-Landau functional leads to investigate the asymptotics of the lowest eigenvalues of Schrödinger operators with magnetic fields $(i h \nabla+\mathbf{A})^{2}$ and Neumann boundary conditions on smooth domains $\Omega$ in $\mathbb{R}^{3}$. Then, near the boundary of $\Omega$, the magnetic field can be approximated by a constant field which makes an angle $\theta \in\left[0, \frac{\pi}{2}\right]$ with the boundary (approximated by the tangent

*IRMAR, ENS Cachan Bretagne, Univ. Rennes 1, CNRS, UEB, av. Robert Schuman, F-35170 Bruz, France, virginie.bonnaillie@bretagne.ens-cachan.fr

†IRMAR, Universite Rennes 1, CNRS, UMR 6625, Campus de Beaulieu, F-35042 Rennes cedex, France, monique.daugeduniv-rennesl.fr, nicolas.popoff@univ-rennesl.fr, nicolas.raymondeuniv-rennesi.fr 
plane). Thus, after a choice of gauge, we are led to investigate the operator with Neumann conditions on the half-space $\mathbb{R}_{+}^{3}=\left\{(r, s, t) \in \mathbb{R}^{3}: t>0\right\}$ :

$$
h^{2} D_{s}^{2}+h^{2} D_{t}^{2}+\left(h D_{r}+t \cos \theta-s \sin \theta\right)^{2},
$$

where $D_{x}$ denotes $-i \partial_{x}$ for any variable $x$. The first step in the study of this operator is a Fourier transform in $r$. If $\theta=0$, we are led to the so-called de Gennes operator on an halfline (see [7]). If $\theta \neq 0$, after a translation in $s$ we are reduced to a Schrödinger operator with an electric potential on the half-plane $\mathbb{R}_{+}^{2}=\left\{(s, t) \in \mathbb{R}^{2}: t>0\right\}$ :

$$
h^{2} D_{s}^{2}+h^{2} D_{t}^{2}+(t \cos \theta-s \sin \theta)^{2} .
$$

After a rescaling, we can reduce to the case $h=1$.

Thus, this is a natural question to wonder how the eigenpairs of this operator behave when $\theta$ goes to 0 (the form domain does not depend continuously on $\theta$ ).

In this paper, we investigate this question and study the exponential concentration of eigenvectors.

\subsection{Discrete and essential spectrum of model operators}

We denote by $x=(s, t)$ the coordinates in $\mathbb{R}^{2}$ and by $\Omega$ the half-plane:

$$
\Omega=\mathbb{R}_{+}^{2}=\left\{x=(s, t) \in \mathbb{R}^{2}, t>0\right\} .
$$

We study the self-adjoint Neumann realization on the half-plane $\Omega$ of the Schrödinger operator $\mathcal{L}_{\theta}$ with potential $V_{\theta}$ :

$$
\mathcal{L}_{\theta}=-\Delta+V_{\theta}=D_{s}^{2}+D_{t}^{2}+V_{\theta}
$$

where $V_{\theta}$ is defined for any $\theta \in\left(0, \frac{\pi}{2}\right)$ by

$$
V_{\theta}: x=(s, t) \in \Omega \longmapsto(t \cos \theta-s \sin \theta)^{2} .
$$

We can notice that $V_{\theta}$ reaches its minimum 0 all along the line $t \cos \theta=s \sin \theta$, which makes the angle $\theta$ with $\partial \Omega$. We denote by $D_{N}\left(\mathcal{L}_{\theta}\right)$ the domain of $\mathcal{L}_{\theta}$ and we consider the associated quadratic form $q_{\theta}$ defined by:

$$
q_{\theta}(u)=\int_{\Omega}\left(|\nabla u|^{2}+V_{\theta}|u|^{2}\right) \mathrm{d} x
$$

whose domain $D\left(q_{\theta}\right)$ is:

$$
D\left(q_{\theta}\right)=\left\{u \in L^{2}(\Omega), \nabla u \in L^{2}(\Omega), \sqrt{V_{\theta}} u \in L^{2}(\Omega)\right\} .
$$

The operator $\mathcal{L}_{\theta}$ is positive. We now recall the min-max principle which links the $n$-th eigenvalue to Rayleigh quotients (see [22, Theorem XIII.1], [23, p. 75]): 
Proposition 1.1 (min-max principle) Let $A$ be a self-adjoint operator that is bounded from below, $q_{A}$ its quadratic form and $D\left(q_{A}\right)$ its form domain. Let us define

$$
\mu_{n}=\sup _{\Psi_{1}, \ldots, \Psi_{n-1} \in D\left(q_{A}\right)} \inf _{\substack{\Psi \in\left[\Psi_{1}, \ldots, \Psi_{n-1}\right]^{\perp} \\ \Psi \in D\left(q_{A}\right),\|\Psi\|=1}} q_{A}(\Psi)=\inf _{\Psi_{1}, \ldots, \Psi_{n} \in D\left(q_{A}\right)} \sup _{\substack{\Psi \in\left[\Psi_{1}, \ldots, \Psi_{n}\right] \\\|\Psi\|=1}} q_{A}(\Psi) .
$$

Then, for each fixed $n$, we have the alternative " $(a)$ or $(b)$ ":

(a) There are $n$ eigenvalues (counted with multiplicity) below the bottom of the essential spectrum, and $\mu_{n}$ is the $n$-th eigenvalue counted with multiplicity;

(b) $\mu_{n}$ is the bottom of the essential spectrum, and in that case $\mu_{n}=\mu_{n+1}=\ldots$ and there are at most $n-1$ eigenvalues (counting multiplicity) below $\mu_{n}$.

Let $\sigma_{n}(\theta)$ denote the $n$-th Rayleigh quotient of $\mathcal{L}_{\theta}$ defined by (1.1). Let $\operatorname{sp}_{\text {dis }}\left(\mathcal{L}_{\theta}\right)$ and $\operatorname{sp}_{\text {ess }}\left(\mathcal{L}_{\theta}\right)$ be its discrete and essential spectrum, respectively. Let us recall some fundamental spectral properties of $\mathcal{L}_{\theta}$ when $\theta \in\left(0, \frac{\pi}{2}\right)$.

It is proved in [11] that $\operatorname{sp}_{\text {ess }}\left(\mathcal{L}_{\theta}\right)=[1,+\infty)$ and that $\theta \mapsto \sigma_{n}(\theta)$ is non decreasing. Moreover, the function $\left(0, \frac{\pi}{2}\right) \ni \theta \mapsto \sigma_{1}(\theta)$ is increasing, and corresponds to a simple eigenvalue $<1$ associated with a positive eigenfunction (see [15, Lemma 3.6]). As a consequence $\theta \mapsto \sigma_{1}(\theta)$ is analytic (see for example [14, Chapter 7]).

Remark 1.2 By an even reflection through the boundary, our problem is equivalent to a problem set on the whole plane $\mathbb{R}^{2}$ with a potential which reaches its minimum on the union of two half-lines: $\mathcal{L}_{\theta}$ has eigenvalues under its essential spectrum if and only if the half-lines are not colinear. It is interesting to note the analogy with quantum wave guides which have eigenvalues below their essential spectrum as soon as their middle fiber has a non zero curvature (see $[8,5])$.

In all our investigations, of fundamental importance is the family of one-dimensional self-adjoint operators $H_{\zeta}, \zeta \in \mathbb{R}$, defined by:

$$
H_{\zeta}=H_{\zeta}\left(t ; D_{t}\right)=D_{t}^{2}+(t-\zeta)^{2}
$$

on their common Neumann domain on the half-line:

$$
\left\{v \in H^{2}\left(\mathbb{R}_{+}\right), t^{2} v \in L^{2}\left(\mathbb{R}_{+}\right), v^{\prime}(0)=0\right\} .
$$

The spectral properties of this family of operators have been studied in [7]. Let us recall some of these. We denote by $\mu(\zeta)$ the lowest eigenvalue of $H_{\zeta}$, and by $v_{\zeta}$ a normalized associated eigenfunction. We have the following limits (see [7, §3]):

$$
\lim _{\zeta \rightarrow+\infty} \mu(\zeta)=1 \quad \text { and } \quad \lim _{\zeta \rightarrow-\infty} \mu(\zeta)=+\infty
$$


Let us also mention that

$$
\mu(\zeta) \underset{\zeta \rightarrow-\infty}{=} \mathcal{O}\left(\zeta^{2}\right)
$$

In addition, the function $\mu$ reaches (non degenerately) its minimum denoted by $\Theta_{0}$ for a unique value $\zeta_{0}$ (as proved in [7, Theorem 4.3]). There holds (see [4] for refined numerical computations):

$$
\zeta_{0}^{2}=\Theta_{0} \quad \text { and } \quad \Theta_{0} \simeq 0.590106125
$$

\subsection{Main results of the paper}

Our results concern exponential decay estimates for eigenvectors of $\mathcal{L}_{\theta}$ and the asymptotic behavior of its eigenvalues in the small angle limit $\theta \rightarrow 0$. All along this paper, $\left(\sigma(\theta), u_{\theta}\right)$ will denote an eigenpair of $\mathcal{L}_{\theta}$ with $\sigma(\theta)<1$. We prove the exponential decay estimates for $u_{\theta}$ stated in the following two theorems, improving the results of [21]. Our first result gives an isotropic exponential decay with a weight of the type $e^{\alpha|x|}$ :

Theorem 1.3 Let $\left(\sigma(\theta), u_{\theta}\right)$ be an eigenpair of $\mathcal{L}_{\theta}$ with $\sigma(\theta)<1$. We have:

$$
\forall \alpha \in(0, \sqrt{1-\sigma(\theta)}), \exists C_{\alpha, \theta}>0, \quad q_{\theta}\left(e^{\alpha|x|} u_{\theta}\right) \leq C_{\alpha, \theta}\left\|u_{\theta}\right\|_{L^{2}(\Omega)}^{2} .
$$

Our second result is an anisotropic decay estimate in the orthogonal direction of the zero set of $V_{\theta}$ :

Theorem 1.4 Let $0<\beta<\frac{1}{2}$. Let $\left(\sigma(\theta), u_{\theta}\right)$ be an eigenpair of $\mathcal{L}_{\theta}$ with $\sigma(\theta)<1$. Then there exists a constant $K(\beta)$ such that

$$
q_{\theta}\left(e^{\beta V_{\theta}} u_{\theta}\right) \leq K(\beta)\left\|u_{\theta}\right\|_{L^{2}(\Omega)}^{2} .
$$

Estimates (1.4) and (1.5) have different performances in different directions: For $\gamma \in$ $[0, \pi]$, let us consider the points

$$
x=r(\cos \gamma, \sin \gamma), \quad r>0,
$$

on the half-line of angle $\gamma$ with $\partial \Omega$. Then

$$
|x|=r \quad \text { and } \quad V_{\theta}(x)=r^{2} \sin ^{2}(\gamma-\theta) .
$$

Thus estimate (1.4) is stronger than (1.5) if $\gamma=\theta$, but weaker as soon as $\gamma \neq \theta$.

Then, we want to analyze the behavior of the eigenvalues below the essential spectrum when $\theta$ goes to zero. In a first step, we prove that the number of such eigenvalues tends to infinity:

Theorem 1.5 We have the following upper bound for the $n$-th eigenvalue $\sigma_{n}(\theta)$ of $\mathcal{L}_{\theta}$ :

$$
\sigma_{n}(\theta) \leq \Theta_{0} \cos \theta+(2 n-1) \sin \theta, \quad \forall n \geq 1 \text {. }
$$


Remark 1.6 If we denote by $n(\theta)$ the number of eigenvalues of $\mathcal{L}_{\theta}$ below the essential spectrum, we have:

$$
n(\theta) \geq \frac{1-\Theta_{0} \cos \theta}{2 \sin \theta}+\frac{1}{2}
$$

In [18, Theorem 2.1], it is proved that this number is finite for any chosen $\theta$.

In a second step, we use semi-classical techniques to prove an expansion in powers of $\theta$ as $\theta \rightarrow 0$ for those eigenvalues:

Theorem 1.7 For all $n \geq 1$, there exists a sequence $\left(\beta_{j, n}\right)_{j \geq 0}$ such that for all $N \geq 1$ and $J \geq 1$, there exist $C_{N, J}>0$ and $\theta_{N}>0$ such that for all $1 \leq n \leq N$ and $0<\theta<\theta_{N}$, $\sigma_{n}(\theta)$ is an eigenvalue and

$$
\left|\sigma_{n}(\theta)-\sum_{j=0}^{J} \beta_{j, n} \theta^{j}\right| \leq C_{N, J} \theta^{J+1} .
$$

Moreover, $\beta_{0, n}=\Theta_{0}$ and $\beta_{1, n}=(2 n-1) \sqrt{\frac{\mu^{\prime \prime}\left(\zeta_{0}\right)}{2}}$.

The proof of this theorem relies on the construction of quasimodes and on projection arguments which show that eigenvectors are close to quasimodes. In this way we prove at the same time a full expansion of eigenvectors associated with the lowest eigenvalues as $\theta \rightarrow 0$, see Section 4.3. A remarkable feature is that the eigenvector expansions do contain half-integer powers of $\theta$, in contrast with the eigenvalue expansions.

\subsection{Organization of the paper}

After the present introduction, we prove in Section 2 the isotropic and anisotropic decay estimate of Theorems 1.3 and 1.4. In Section 3, we prove Theorem 1.5, which shows that the number of eigenvalues below 1 tends to infinity as the angle $\theta$ tends to 0 . We also prove that eigenvalues densify on the whole interval $\left[\Theta_{0}, 1\right]$ when $\theta \rightarrow 0$. Section 4 is devoted to the proof of Theorem 4.1 which immediately implies Theorem 1.7.

In Section 5 we present a series of computations of eigenpairs performed with the finite element library MÉLINA [16]. They illustrate the anisotropic exponential decay of eigenvectors, and also clearly display the four term asymptotic expansion for the $n$-th eigenvalue of $\mathcal{L}_{\theta}$ as $\theta \rightarrow 0$ :

$$
\sigma_{n}(\theta)=\Theta_{0}+(2 n-1) a_{1} \theta-a_{2, n} \theta^{2}-a_{3, n} \theta^{3}+\mathcal{O}\left(\theta^{3}\right),
$$

where $a_{1} \simeq 0.7651882$ and $a_{2, n}, a_{3, n}$ are some positive coefficients. This expansion is coherent with (1.8), all the more since $a_{1}$ coincides with the 7-digit numerical approximation of $\sqrt{\mu^{\prime \prime}\left(\zeta_{0}\right) / 2}$ according to the 1D computations presented in [4]. In addition, for small angles $\theta$, the eigenvectors show their resemblance with the quasimodes constructed in tensor product form, cf. Section 4.3 and Figures 8-10. 


\section{Exponential decay of eigenvectors}

The aim of this section is to prove Theorems 1.3 and 1.4. For that purpose, we need to recall some ingredients in order to implement the so-called "Agmon's estimates". These estimates are related to the Agmon distance the main properties of which can be found in [12] (see also [10, §3.2]).

\subsection{Preliminaries}

Here we recall a few classical identities due to Agmon. There are consequences of the "IMS" formula and can be found in [2] (see also [6] and [20] for the same kind of applications).

Lemma 2.1 Let $u \in D_{N}\left(\mathcal{L}_{\theta}\right)$ and $\Phi$ be a bounded and uniform Lipschitz function defined on $\Omega$. Then we have

$$
\left\langle\mathcal{L}_{\theta} u, e^{2 \Phi} u\right\rangle=q_{\theta}\left(e^{\Phi} u\right)-\left\||\nabla \Phi| e^{\Phi} u\right\|_{L^{2}(\Omega)}^{2}
$$

Taking $u=u_{\theta}$ in Lemma 2.1, we get the obvious corollary:

Corollary 2.2 Let $\left(\sigma(\theta), u_{\theta}\right)$ be an eigenpair for $\mathcal{L}_{\theta}$ and $\Phi$ be a bounded and uniform Lipschitz function defined on $\Omega$. We have the following identities:

$$
\begin{gathered}
\int_{\Omega}\left(\sigma(\theta)+|\nabla \Phi|^{2}\right) e^{2 \Phi}\left|u_{\theta}\right|^{2}=q_{\theta}\left(e^{\Phi} u_{\theta}\right) \\
\int_{\Omega}\left|\nabla\left(e^{\Phi} u_{\theta}\right)\right|^{2}+\int_{\Omega}\left(V_{\theta}-\sigma(\theta)-|\nabla \Phi|^{2}\right) e^{2 \Phi}\left|u_{\theta}\right|^{2}=0 .
\end{gathered}
$$

Let $\left(\Omega^{+}, \Omega^{-}\right)$be a partition of $\Omega: \Omega=\Omega^{+} \cup \Omega^{-}$with $\Omega^{+} \cap \Omega^{-}=\emptyset$, then we have

$$
\int_{\Omega^{+}}\left(V_{\theta}-\sigma(\theta)-|\nabla \Phi|^{2}\right) e^{2 \Phi}\left|u_{\theta}\right|^{2} \leq\left.\left.\sup _{\Omega^{-}}\left|V_{\theta}-\sigma(\theta)-\right| \nabla \Phi\right|^{2}\left|\int_{\Omega^{-}} e^{2 \Phi}\right| u_{\theta}\right|^{2} .
$$

In order to satisfy the hypotheses of this corollary, we will need to perform a partition of unity. This is the aim of the following two lemmas to explain how to deal with such a partition.

Lemma 2.3 Let $\chi \in C_{0}^{\infty}(\bar{\Omega})$ and $u \in D\left(q_{\theta}\right)$, then

$$
q_{\theta}(\chi u)=\int_{\Omega}|\chi|^{2}\left(|\nabla u|^{2}+V_{\theta}|u|^{2}\right)+\frac{1}{2} \int_{\Omega} \nabla|\chi|^{2} \nabla|u|^{2}+\int_{\Omega}|\nabla \chi|^{2}|u|^{2} .
$$

If we suppose moreover that $u \in D_{N}\left(\mathcal{L}_{\theta}\right)$, we have:

$$
q_{\theta}(\chi u)=\left\langle\chi^{2} \mathcal{L}_{\theta} u, u\right\rangle+\||\nabla \chi| u\|_{L^{2}(\Omega)}^{2} .
$$


Lemma 2.4 Let $\left(\chi_{i}\right)_{i}$ be a finite regular partition of unity with $\sum_{i} \chi_{i}^{2}=1$. Then for all $u \in D\left(q_{\theta}\right)$,

$$
\sum_{i} q_{\theta}\left(\chi_{i} u\right)=q_{\theta}(u)+\int_{\Omega} \sum_{i}\left|\nabla \chi_{i}\right|^{2}|u|^{2} .
$$

\subsection{Isotropic decay of the eigenvectors}

This subsection is devoted to the proof of Theorem 1.3.

PRELIMINARIES. Let $\left(\chi_{1}, \chi_{2}\right)$ be a partition of unity on $\mathbb{R}^{+}$with $\chi_{1}^{2}+\chi_{2}^{2}=1$ and:

$$
\left\{\begin{array}{l}
0 \leq \chi_{1} \leq 1, \quad \chi_{1}(r)=1 \text { if } r \leq 1, \text { and } 0 \text { if } r \geq 2, \\
0 \leq \chi_{2} \leq 1, \quad \chi_{2}(r)=0 \text { if } r \leq 1, \text { and } 1 \text { if } r \geq 2 .
\end{array}\right.
$$

We define

$$
\chi_{1}^{R}(x)=\chi_{1}\left(\frac{|x|}{R}\right) \quad \text { and } \quad \chi_{2}^{R}(x)=\chi_{2}\left(\frac{|x|}{R}\right) .
$$

We have $\nabla \chi_{j}^{R}(x)=\frac{1}{R} \nabla \chi_{j}\left(\frac{x}{R}\right)$. Thus we deduce:

$$
\exists C>0, \forall j=1,2, \forall x \in \Omega, \quad\left|\nabla \chi_{j}^{R}(x)\right| \leq \frac{C}{R} .
$$

Let us fix $\alpha>0$. As Agmon's distance, we choose the function:

$$
\Phi(s, t)=\alpha \sqrt{s^{2}+t^{2}}=\alpha|x| .
$$

It clearly satisfies $|\nabla \Phi|^{2}=\alpha^{2}$. We do not know yet that $e^{\Phi} u_{\theta} \in D\left(q_{\theta}\right)$. This is the reason why we use a cut-off function in order to use the Corollary 2.2. We define for $k \in \mathbb{N}$ :

$$
\begin{cases}\Phi_{k}(x)=\alpha|x| & \text { if }|x| \leq k \\ \Phi_{k}(x)=\alpha(2 k-|x|) & \text { if } k \leq|x| \leq 2 k \\ \Phi_{k}(x)=0 & \text { if }|x| \geq 2 k\end{cases}
$$

We have:

$$
\begin{cases}\left|\nabla \Phi_{k}\right|^{2}=|\nabla \Phi|^{2}=\alpha^{2} & \text { if }|x| \leq 2 k \\ \left|\nabla \Phi_{k}\right|^{2}=0 & \text { if }|x|>2 k .\end{cases}
$$

FIRST STEP. Using (2.2) and (2.7) we have:

$$
\int_{\Omega}\left(\sigma(\theta)+\left|\nabla \Phi_{k}\right|^{2}\right) e^{2 \Phi_{k}}\left|u_{\theta}\right|^{2}=\sum_{j=1}^{2} q_{\theta}\left(\chi_{j}^{R} e^{\Phi_{k}} u_{\theta}\right)-\sum_{j=1}^{2} \int_{\Omega}\left|\nabla \chi_{j}^{R}\right|^{2} e^{2 \Phi_{k}}\left|u_{\theta}\right|^{2} .
$$

Let us choose $\varepsilon \in(0,1-\sigma(\theta))$ and

$$
\alpha=\sqrt{1-\varepsilon-\sigma(\theta)} .
$$


Thus, we have:

$$
\sigma(\theta)+|\nabla \Phi|^{2}=1-\varepsilon
$$

It follows that:

$$
\int_{\Omega}\left(\sigma(\theta)+\left|\nabla \Phi_{k}\right|^{2}\right) e^{2 \Phi_{k}}\left|u_{\theta}\right|^{2}=(1-\varepsilon)\left\|e^{\Phi_{k}} u_{\theta}\right\|_{L^{2}(\Omega)}^{2}+(\sigma(\theta)-1+\varepsilon) \int_{|x|>2 k}\left|u_{\theta}\right|^{2} \mathrm{~d} x
$$

We choose $R>0$ such that

$$
\frac{C^{2}}{R^{2}} \leq \frac{\varepsilon}{4}
$$

where $C$ is the constant appearing in (2.9). Hence we get:

$$
\sum_{j=1}^{2} \int_{\Omega}\left|\nabla \chi_{j}^{R}\right|^{2}\left|e^{\Phi_{k}} u_{\theta}\right|^{2} \leq \frac{\varepsilon}{2}\left\|e^{\Phi_{k}} u_{\theta}\right\|_{L^{2}(\Omega)}^{2}
$$

Relations (2.11), (2.14) and (2.16) provide:

$$
\begin{aligned}
\frac{\varepsilon}{2}\left\|e^{\Phi_{k}} u_{\theta}\right\|_{L^{2}(\Omega)}^{2} & \leq\left\|e^{\Phi_{k}} u_{\theta}\right\|_{L^{2}(\Omega)}^{2}-\sum_{j=1}^{2} q_{\theta}\left(\chi_{j}^{R} e^{\Phi_{k}} u_{\theta}\right)+(\sigma(\theta)-1+\varepsilon) \int_{|x|>2 k}\left|u_{\theta}\right|^{2} \mathrm{~d} x \\
& \leq\left\|e^{\Phi_{k}} u_{\theta}\right\|_{L^{2}(\Omega)}^{2}-q_{\theta}\left(\chi_{2}^{R} e^{\Phi_{k}} u_{\theta}\right) .
\end{aligned}
$$

SECOND STEP. In order to bound from below the energy "far from the origin" $q_{\theta}\left(\chi_{2}^{R} e^{\Phi_{k}} u_{\theta}\right)$ we introduce a classical notation attached to Persson's lemma:

$$
\Sigma\left(\mathcal{L}_{\theta}, r\right)=\inf \left\{q_{\theta}(u),\|u\|_{L^{2}(\Omega)}=1, u \in C_{0}^{\infty}\left(\bar{\Omega} \cap \complement B_{r}\right)\right\}
$$

where $B_{r}$ denotes the ball of radius $r$ centered at 0 , and $\complement B_{r}$ its complement. It results from Persson's lemma (see [19]) that the limit of $\Sigma\left(\mathcal{L}_{\theta}, r\right)$ as $r \rightarrow+\infty$ equals the bottom of the essential spectrum of $\mathcal{L}_{\theta}$, thus 1 :

$$
\lim _{r \rightarrow+\infty} \Sigma\left(\mathcal{L}_{\theta}, r\right)=1
$$

We have:

$$
\frac{q_{\theta}\left(\chi_{2}^{R} e^{\Phi_{k}} u_{\theta}\right)}{\left\|\chi_{2}^{R} e^{\Phi_{k}} u_{\theta}\right\|_{L^{2}(\Omega)}^{2}} \geq \Sigma\left(\mathcal{L}_{\theta}, R\right)
$$

and so:

$$
q_{\theta}\left(\chi_{2}^{R} e^{\Phi_{k}} u_{\theta}\right) \geq \Sigma\left(\mathcal{L}_{\theta}, R\right) \int_{|x|>2 R} e^{2 \Phi_{k}}\left|u_{\theta}\right|^{2} \mathrm{~d} x
$$

Using (2.17), we get:

$$
\frac{\varepsilon}{2}\left\|e^{\Phi_{k}} u_{\theta}\right\|_{L^{2}(\Omega)}^{2} \leq \int_{|x|<2 R} e^{2 \Phi_{k}}\left|u_{\theta}\right|^{2} \mathrm{~d} x+\left(1-\Sigma\left(\mathcal{L}_{\theta}, R\right)\right) \int_{|x|>2 R} e^{2 \Phi_{k}}\left|u_{\theta}\right|^{2} \mathrm{~d} x .
$$


Using (2.18), we can choose $R$ large enough such that, besides (2.15):

$$
1-\Sigma\left(\mathcal{L}_{\theta}, R\right)<\frac{\varepsilon}{4} .
$$

We deduce:

$$
\frac{\varepsilon}{4} \int_{\Omega} e^{2 \Phi_{k}}\left|u_{\theta}\right|^{2} \leq \int_{|x|<2 R} e^{2 \Phi_{k}}\left|u_{\theta}\right|^{2} \mathrm{~d} x .
$$

We finally get:

$$
\forall k \in \mathbb{N}, \quad\left\|e^{\Phi_{k}} u_{\theta}\right\|_{L^{2}(\Omega)}^{2} \leq \frac{4}{\varepsilon} e^{4 \alpha R}\left\|u_{\theta}\right\|_{L^{2}(\Omega)}^{2} .
$$

Conclusion. However, $\left|e^{\Phi_{k}} u_{\theta}\right|$ converges pointwise to $\left|e^{\Phi} u_{\theta}\right|$ as $k$ goes to infinity. It follows from Fatou's lemma that $e^{\Phi} u_{\theta} \in L^{2}(\Omega)$. The conclusion comes from:

$$
\left\|\nabla\left(e^{\Phi} u_{\theta}\right)\right\|_{L^{2}(\Omega)}^{2}+\left\|V_{\theta} e^{\Phi} u_{\theta}\right\|_{L^{2}(\Omega)}^{2}=(1-\varepsilon)\left\|e^{\Phi} u_{\theta}\right\|_{L^{2}(\Omega)}^{2},
$$

as a direct consequence of (2.3) and (2.13).

Remark 2.5 This proof is the key point in order to prove that $u_{\theta}$ is in the Schwartz's class, see [21].

Examining the arguments of this proof, we can see that $\alpha$ and the constant $C_{\alpha, \theta}$ can be chosen uniformly in any closed interval $\left[\theta_{0}, \theta_{1}\right]$ with $\theta_{0}>0$ and $\theta_{1}<\frac{\pi}{2}$. Since $\sigma(\theta) \rightarrow 1$ as $\theta \rightarrow \frac{\pi}{2}$, it is impossible to obtain uniform estimates as $\theta \rightarrow \frac{\pi}{2}$. When $\theta \rightarrow 0$, there is no valid uniform estimates in the tangential variable $s$. However, considering only a dependence with respect to $t$, we get a uniform control in $\theta$ :

Proposition 2.6 Let $\eta<1$. There exist $C>0$ and $\gamma>0$ such that for any eigenpair $\left(\sigma(\theta), u_{\theta}\right)$ of $\mathcal{L}_{\theta}$ with $\sigma(\theta) \leq \eta$, there holds

$$
\int_{\Omega} e^{2 \gamma t}\left|u_{\theta}\right|^{2} \mathrm{~d} s \mathrm{~d} t \leq C\left\|u_{\theta}\right\|_{L^{2}(\Omega)}^{2} .
$$

Proof: The proof is similar as for Theorem 1.3. We choose $\Phi=\gamma t$ and instead (2.8) we use the partition of unity $\left(\chi_{j}^{R}\right)$ with respect to $t$ :

$$
\chi_{1}^{R}(x)=\chi_{1}\left(\frac{t}{R}\right) \quad \text { and } \quad \chi_{2}^{R}(x)=\chi_{2}\left(\frac{t}{R}\right) .
$$

The first step of the proof goes the same way with

$$
\varepsilon \in(0,1-\eta),
$$

and the key point of the second step is then the following lower bound which replaces (2.20):

$$
q_{\theta}\left(\chi_{2}^{R} e^{\Phi} u_{\theta}\right) \geq\left\|\chi_{2}^{R} e^{\Phi} u_{\theta}\right\|_{L^{2}(\Omega)}^{2} .
$$

This inequality is a consequence of the fact that the support of $\chi_{2}^{R}$ is now far from the boundary of $\Omega$ and that the bottom of the spectrum of the self-adjoint realization on $\mathbb{R}^{2}$ of $D_{t}^{2}+D_{s}^{2}+V_{\theta}$ is 1 . Thus, as $\varepsilon$ is set and the size of $R$ does not depend anymore on $\theta$ for this choice of $\Phi$, we get that the upper bound in (2.21) is independent from $\theta$. 


\subsection{Anisotropic decay from the minimum of the potential}

In this section we prove the decay of $u_{\theta}$ away from the minimum of $V_{\theta}$ stated in Theorem 1.4. Let $\delta \in(0,1)$. Following [1], we introduce the function associated with Agmon's geodesics:

$$
\Phi(x)=(1-\delta) \int_{\sqrt{\sigma(\theta)}}^{\sqrt{V_{\theta}(x)}} \sqrt{\left(l^{2}-\sigma(\theta)\right)_{+}} \mathrm{d} l,
$$

where $f_{+}$denotes the positive part of a function $f$. Let us notice that if we define the function

$$
g(d)=\int_{\sqrt{\sigma(\theta)}}^{d} \sqrt{\left(l^{2}-\sigma(\theta)\right)_{+}} \mathrm{d} l
$$

we have

$$
\Phi(x)=(1-\delta) g\left(\sqrt{V_{\theta}(x)}\right) .
$$

It is an elementary computation to check that we have (uniformly in $\theta$ ):

$$
g(d) \underset{d \rightarrow+\infty}{=} \frac{d^{2}}{2}+\mathcal{O}(\ln d) \quad \text { and } \quad g^{\prime}(d) \underset{d \rightarrow+\infty}{=} d+\mathcal{O}\left(d^{-1}\right)
$$

So Theorem 1.4 holds if and only if $q_{\theta}\left(e^{\Phi} u_{\theta}\right)$ is bounded uniformly in $\theta$ for all $\delta \in(0,1)$.

Let us prove this. We choose $\delta \in(0,1)$. By construction of $\Phi$, we have:

$$
|\nabla \Phi|^{2}=(1-\delta)^{2}\left(V_{\theta}-\sigma(\theta)\right)_{+}
$$

Let $\eta>0$, we define a partition of unity for $\Omega$ :

$$
A_{\eta}^{+}=\left\{(s, t) \in \Omega, V_{\theta}(s, t)-\sigma(\theta)>\eta\right\} \quad \text { and } \quad A_{\eta}^{-}=\left\{(s, t) \in \Omega, V_{\theta}(s, t)-\sigma(\theta) \leq \eta\right\} \text {. }
$$

On $A_{\eta}^{+}$, we have:

$$
V_{\theta}-\sigma(\theta)-|\nabla \Phi|^{2}=\left(V_{\theta}-\sigma(\theta)\right)\left(2 \delta-\delta^{2}\right)>\eta\left(2 \delta-\delta^{2}\right)
$$

Similarly, we have on $A_{\eta}^{-}$:

$$
\left.\left|V_{\theta}-\sigma(\theta)-\right| \nabla \Phi\right|^{2} \mid= \begin{cases}\sigma(\theta)-V_{\theta} & \text { if } V_{\theta}<\sigma(\theta) \\ \left(V_{\theta}-\sigma(\theta)\right)\left(2 \delta-\delta^{2}\right) & \text { if not. }\end{cases}
$$

Let us assume that

$$
0<\eta\left(2 \delta-\delta^{2}\right) \leq \Theta_{0}<\sigma(\theta) .
$$

Then, we have the following upper bound:

$$
\left.\sup _{A_{\eta}^{-}}\left|V_{\theta}-\sigma(\theta)-\right| \nabla \Phi\right|^{2} \mid \leq \sigma(\theta) .
$$


We now combine (2.4), (2.28) and (2.30) in order to get:

$$
\eta\left(2 \delta-\delta^{2}\right) \int_{A_{\eta}^{+}} e^{2 \Phi}\left|u_{\theta}\right|^{2} \leq \int_{A_{\eta}^{+}}\left(V_{\theta}-\sigma(\theta)-|\nabla \Phi|^{2}\right) e^{2 \Phi}\left|u_{\theta}\right|^{2} \leq \sigma(\theta) \int_{A_{\eta}^{-}} e^{2 \Phi}\left|u_{\theta}\right|^{2} .
$$

Since $\left\|u_{\theta}\right\|_{L^{2}(\Omega)}=1$ and that $\Phi$ is maximal on the boundary of $A_{\eta}^{-}$, we get:

$$
\left\|e^{\Phi} u_{\theta}\right\|_{L^{2}(\Omega)} \leq\left(\frac{\sigma(\theta)}{\eta\left(2 \delta-\delta^{2}\right)}+1\right) \exp \int_{\sqrt{\sigma(\theta)}}^{\sqrt{\sigma(\theta)+\eta}}(1-\delta) \sqrt{l^{2}-\sigma(\theta)} \mathrm{d} l .
$$

We denote by $K(\eta, \delta, \sigma(\theta))$ the right hand side of the last inequality. If we fix $\delta>0$, the function

$$
\begin{aligned}
\mathbb{R}_{+} \times\left[\Theta_{0}, 1\right] & \rightarrow \mathbb{R} \\
(\eta, \sigma) & \rightarrow K(\eta, \delta, \sigma)
\end{aligned}
$$

is clearly positive and continuous. We notice that:

$$
\lim _{\eta \rightarrow 0} K(\eta, \delta, \sigma)=+\infty
$$

Recall that we assume the condition on $\eta$ given by (2.29). We introduce the interval $I(\delta)=$ $\left(0, \frac{\Theta_{0}}{2 \delta-\delta^{2}}\right]$. This allows us to define the positive constant

$$
K_{0}(\delta)=\max _{\sigma \in\left[\Theta_{0}, 1\right]} \min _{\eta \in I(\delta)} K(\eta, \delta, \sigma) .
$$

The minimum is achieved for a $\eta_{0} \in I(\delta)$. Choosing this $\eta_{0}$, we deduce from (2.32):

$$
\left\|e^{\Phi} u_{\theta}\right\|_{L^{2}(\Omega)} \leq K_{0}(\delta) .
$$

If we define

$$
\tilde{\Phi}(x)=\left(1-\frac{\delta}{2}\right) \int_{\sqrt{\sigma(\theta)}}^{\sqrt{V_{\theta}(x)}} \sqrt{\left(l^{2}-\sigma(\theta)\right)_{+}} \mathrm{d} l
$$

we have

$$
\left\|e^{\tilde{\Phi}} u_{\theta}\right\|_{L^{2}(\Omega)} \leq K_{0}\left(\frac{\delta}{2}\right)
$$

Because of (2.26), we have easily

$$
\exists K_{1}(\delta)>0, \forall d>0,\left|d e^{-\frac{\delta}{2} g(d)}\right|<K_{1}(\delta) .
$$

We notice that $\sqrt{V_{\theta}} e^{\Phi-\tilde{\Phi}}=\sqrt{V_{\theta}} e^{-\frac{\delta}{2} g\left(\sqrt{V_{\theta}}\right)}$ and with (2.34), we deduce:

$$
\exists K_{1}(\delta)>0, \quad\left\|\sqrt{V_{\theta}} e^{\Phi-\tilde{\Phi}}\right\|_{L^{\infty}(\Omega)} \leq K_{1}(\delta) .
$$


Therefore, we have:

$$
\left\|\sqrt{V_{\theta}} e^{\Phi} u_{\theta}\right\|_{L^{2}(\Omega)} \leq\left\|\sqrt{V_{\theta}} e^{\Phi-\tilde{\Phi}}\right\|_{L^{\infty}(\Omega)}\left\|e^{\tilde{\Phi}} u_{\theta}\right\|_{L^{2}(\Omega)},
$$

and finally, with a new constant $K_{2}(\delta)$ :

$$
\left\|\sqrt{V_{\theta}} e^{\Phi} u_{\theta}\right\|_{L^{2}(\Omega)} \leq K_{2}(\delta) .
$$

With the definition of $\Phi$, we also get:

$$
\left\||\nabla \Phi| e^{\Phi} u_{\theta}\right\|_{L^{2}(\Omega)} \leq K_{3}(\delta) .
$$

Using (2.1), we finally obtain

$$
q_{\theta}\left(e^{\Phi} u_{\theta}\right)=\left\||\nabla \Phi| e^{\Phi} u_{\theta}\right\|_{L^{2}(\Omega)}^{2}+\sigma(\theta)\left\|e^{\Phi} u_{\theta}\right\|_{L^{2}(\Omega)}^{2} \leq K(\delta) .
$$

\section{Densification of the spectrum for small angles}

In this section, we investigate the behavior of the eigenvalues below 1 .

\subsection{An upper bound}

In this section, we give the proof of Theorem 1.5. In order to get the announced upper bound, we will construct a family of quasimodes and use the min-max principle.

Let us introduce first some tools from spectral theory of self-adjoint operators (see for example [22]). We denote by $b_{\theta}(u)$ the Rayleigh quotient associated with a function $u$ for $\mathcal{L}_{\theta}:$

$$
\forall u \in D\left(q_{\theta}\right) \backslash\{0\}, \quad b_{\theta}(u)=\frac{q_{\theta}(u)}{\|u\|_{L^{2}(\Omega)}^{2}} .
$$

The bilinear form associated with $q_{\theta}$ is defined on the form domain by:

$$
a_{\theta}(u, v)=\int_{\Omega}\left(D_{t} u D_{t} v+D_{s} u D_{s} v+V_{\theta} u v\right) \mathrm{d} x .
$$

Lemma 3.1 Let $v_{\zeta_{0}}$ be a normalized eigenvector associated with the first eigenvalue $\Theta_{0}$ of the operator $H_{\zeta_{0}}$ (cf. (1.2) and the properties recalled there), and let $\psi_{n}$ be the $n$-th Hermite function with the "physicists" convention. We recall that:

$$
\forall n \geq 0, \forall x \in \mathbb{R}, \quad-\psi_{n}^{\prime \prime}(x)+x^{2} \psi_{n}(x)=(2 n+1) \psi_{n}(x) .
$$

We define the normalized function $\widetilde{u}_{n, \theta}$ by:

$$
\widetilde{u}_{n, \theta}(s, t)=(\cos \theta \sin \theta)^{\frac{1}{4}} v_{\zeta_{0}}(t \sqrt{\cos \theta}) \psi_{n}\left(s \sqrt{\sin \theta}-\frac{\zeta_{0}}{\sqrt{\tan \theta}}\right) .
$$

Then we have:

$$
\forall n \in \mathbb{N}, \forall \theta \in\left(0, \frac{\pi}{2}\right), \quad b_{\theta}\left(\widetilde{u}_{n, \theta}\right)=\Theta_{0} \cos \theta+(2 n+1) \sin \theta .
$$


Proof: The function $\widetilde{u}_{n, \theta}$ is clearly in the form domain $D\left(q_{\theta}\right)$. We are going to estimate $q_{\theta}\left(\widetilde{u}_{n, \theta}\right)$. Let us make the following rescaling and translation:

$$
\left\{\begin{array}{l}
y=s \sqrt{\sin \theta}-\frac{\zeta_{0}}{\sqrt{\tan \theta}}, \\
z=t \sqrt{\cos \theta} .
\end{array}\right.
$$

Then

$$
\begin{aligned}
q_{\theta}\left(\widetilde{u}_{n, \theta}\right)= & \int_{\Omega}\left(\cos \theta\left|v_{\zeta_{0}}^{\prime}(z) \psi_{n}(y)\right|^{2}+\sin \theta\left|v_{\zeta_{0}}(z) \psi_{n}^{\prime}(y)\right|^{2}\right. \\
& \left.\quad+\left(z \sqrt{\cos \theta}-y \sqrt{\sin \theta}-\zeta_{0} \sqrt{\cos \theta}\right)^{2}\left|v_{\zeta_{0}}(z) \psi_{n}(y)\right|^{2}\right) \mathrm{d} y \mathrm{~d} z \\
= & \cos \theta \int_{\Omega}\left(\left|v_{\zeta_{0}}^{\prime}(z) \psi_{n}(y)\right|^{2}+\left(z-\zeta_{0}\right)^{2}\left|v_{\zeta_{0}}(z) \psi_{n}(y)\right|^{2}\right) \mathrm{d} y \mathrm{~d} z \\
& +\sin \theta \int_{\Omega}\left(\left|v_{\zeta_{0}}(z) \psi_{n}^{\prime}(y)\right|^{2}+y^{2}\left|v_{\zeta_{0}}(z) \psi_{n}(y)\right|^{2}\right) \mathrm{d} y \mathrm{~d} z \\
& -2 \sqrt{\sin \theta} \sqrt{\cos \theta} \int_{\Omega} y\left(z-\zeta_{0}\right)\left|v_{\zeta_{0}}(z) \psi_{n}(y)\right|^{2} \mathrm{~d} y \mathrm{~d} z .
\end{aligned}
$$

We have the following relations:

$$
\begin{aligned}
\int_{\mathbb{R}_{+}}\left|v_{\zeta_{0}}^{\prime}(z)\right|^{2}+\left(z-\zeta_{0}\right)^{2}\left|v_{\zeta_{0}}(z)\right|^{2} \mathrm{~d} z & =\Theta_{0}, \\
\int_{\mathbb{R}_{+}}\left(z-\zeta_{0}\right)\left|v_{\zeta_{0}}(z)\right|^{2} \mathrm{~d} z & =0 \\
\int_{\mathbb{R}}\left|\psi_{n}^{\prime}(y)\right|^{2}+y^{2}\left|\psi_{n}(y)\right|^{2} \mathrm{~d} y & =2 n+1,
\end{aligned}
$$

where (3.5) is a direct consequence of the Feynman-Hellman formula (see [13] and also Section 4.1). Thus we have

$$
q_{\theta}\left(\widetilde{u}_{n, \theta}\right)=\Theta_{0} \cos \theta\left\|\psi_{n}\right\|_{L^{2}(\mathbb{R})}^{2}+(2 n+1) \sin \theta\left\|v_{\zeta_{0}}\right\|_{L^{2}\left(\mathbb{R}_{+}\right)}^{2} .
$$

Since $v_{\zeta_{0}}$ and $\psi_{n}$ are normalized, and $\left\|\widetilde{u}_{n, \theta}\right\|_{L^{2}(\Omega)}^{2}=1$, we deduce (3.2).

Lemma 3.2 The functions $\widetilde{u}_{n, \theta}, n \geq 0$, are orthogonal for the bilinear form $a_{\theta}$.

Proof: Let $n \neq m$ be two integers. We recall that $\int_{\mathbb{R}} \psi_{n} \psi_{m}=0$. As in the proof of Lemma 3.1, we have:

$$
\begin{aligned}
a_{\theta}\left(\widetilde{u}_{n, \theta}, \widetilde{u}_{m, \theta}\right)= & \Theta_{0} \cos \theta \int_{\mathbb{R}} \psi_{n}(y) \psi_{m}(y) \mathrm{d} y \\
& +\sin \theta \int_{\mathbb{R}} \psi_{n}^{\prime}(y) \psi_{m}^{\prime}(y)+y^{2} \psi_{n}(y) \psi_{m}(y) \mathrm{d} y \\
& -2 \sqrt{\sin \theta} \sqrt{\cos \theta} \int_{\Omega} y\left(z-\zeta_{0}\right) \psi_{n}(y) \psi_{m}(y)\left|v_{\zeta_{0}}(z)\right|^{2} \mathrm{~d} y \mathrm{~d} z .
\end{aligned}
$$


For the second term, we make an integration by parts:

$$
\begin{aligned}
\int_{\mathbb{R}} \psi_{n}^{\prime}(y) \psi_{m}^{\prime}(y) \mathrm{d} y & =\int_{\mathbb{R}}-\psi_{n}^{\prime \prime}(y) \psi_{m}(y) \mathrm{d} y \\
& =\int_{\mathbb{R}}\left(2 n+1-y^{2}\right) \psi_{n}(y) \psi_{m}(y) \mathrm{d} y \\
& =-\int_{\mathbb{R}} y^{2} \psi_{n}(y) \psi_{m}(y) \mathrm{d} y
\end{aligned}
$$

Since the other terms are clearly equal to 0 , we have $a_{\theta}\left(\widetilde{u}_{n, \theta}, \widetilde{u}_{m, \theta}\right)=0$.

Combining Lemmas 3.1 and 3.2, we deduce Theorem 1.5: Indeed, we only have to apply the min-max principle with the functions $\left(\tilde{u}_{n, \theta}\right)_{n \in \mathbb{N}}$ which are orthogonal for the bilinear form associated with $\mathcal{L}_{\theta}$.

We now show that the eigenvalues get dense in $\left[\Theta_{0}, 1\right]$.

\subsection{Spectrum density}

Proposition 3.3 Let $\zeta>0$ and $n$ be an integer such that

$$
\mu(\zeta) \cos \theta+(2 n+1) \sin \theta<1 .
$$

Then, there exist an eigenvalue $\lambda$ of $\mathcal{L}_{\theta}$ and a constant $C_{\zeta}>0$ such that:

$$
|\mu(\zeta) \cos \theta+(2 n+1) \sin \theta-\lambda| \leq C_{\zeta} \sqrt{2 \cos \theta \sin \theta} \sqrt{n^{2}+1}
$$

Proof: In the same way as previously, we define the functions:

$$
\widetilde{u}_{n, \theta ; \zeta}(s, t)=(\cos \theta \sin \theta)^{\frac{1}{4}} v_{\zeta}(t \sqrt{\cos \theta}) \psi_{n}\left(s \sqrt{\sin \theta}-\frac{\zeta}{\sqrt{\tan \theta}}\right) .
$$

where $v_{\zeta}$ is the normalized eigenfunction associated with the first eigenvalue $\mu(\zeta)$ of $H_{\zeta}$ (cf. $\S 1.1$ ). These functions are clearly in the form domain of $\mathcal{L}_{\theta}$. We have:

$$
\begin{aligned}
& D_{t}^{2} \widetilde{u}_{n, \theta ; \zeta}(s, t)=\cos \theta\left(\mu(\zeta)-(t \sqrt{\cos \theta}-\zeta)^{2}\right) \widetilde{u}_{n, \theta ; \zeta}(s, t) \\
& D_{s}^{2} \widetilde{u}_{n, \theta ; \zeta}(s, t)=\sin \theta\left(2 n+1-\left(s \sqrt{\sin \theta}-\frac{\zeta}{\sqrt{\tan \theta}}\right)^{2}\right) \widetilde{u}_{n, \theta ; \zeta}(s, t) .
\end{aligned}
$$

We deduce

$$
\begin{aligned}
\mathcal{L}_{\theta} \widetilde{u}_{n, \theta ; \zeta}-(\mu(\zeta) \cos \theta+ & (2 n+1) \sin \theta) \widetilde{u}_{n, \theta ; \zeta}= \\
& 2(\cos \theta \sin \theta)^{\frac{1}{2}}\left(\frac{\zeta}{\sqrt{\tan \theta}}-s \sqrt{\sin \theta}\right)(t \sqrt{\cos \theta}-\zeta) \widetilde{u}_{n, \theta ; \zeta}
\end{aligned}
$$


Thus, noticing that $\left\|\widetilde{u}_{n, \theta ; \zeta}\right\|_{L^{2}(\Omega)}=1$, we get:

$$
\begin{aligned}
& \left\|\mathcal{L}_{\theta} \widetilde{u}_{n, \theta ; \zeta}-(\mu(\zeta) \cos \theta+(2 n+1) \sin \theta) \widetilde{u}_{n, \theta ; \zeta}\right\|_{L^{2}(\Omega)}= \\
& \quad 2(\cos \theta \sin \theta)^{\frac{1}{2}}\left\|(t-\zeta) v_{\zeta}(t)\right\|_{L^{2}\left(\mathbb{R}_{+}\right)}\left\|s \psi_{n}(s)\right\|_{L^{2}(\mathbb{R})} .
\end{aligned}
$$

It is well known that

$$
\int_{\mathbb{R}} s^{2} \psi_{n}^{2}(s) \mathrm{d} s=\frac{n^{2}+1}{2},
$$

and if we define $C_{\zeta}=\left\|(t-\zeta) v_{\zeta}\right\|_{L^{2}\left(\mathbb{R}_{+}\right)}$, we can conclude with the spectral theorem.

We can notice that the right part of (3.8) goes to infinity as $n$ gets large, so the previous proposition is useless if $\theta$ is fixed and $n$ goes to infinity. However we have the following proposition:

Proposition 3.4 We have the densification result:

$$
\forall \lambda_{0} \in\left(\Theta_{0}, 1\right), \forall \varepsilon>0, \exists \theta_{*} \in\left(0, \frac{\pi}{2}\right), \forall \theta \in\left(0, \theta_{*}\right], \quad \operatorname{dist}\left(\operatorname{sp}_{\text {dis }}\left(\mathcal{L}_{\theta}\right), \lambda_{0}\right)<\varepsilon .
$$

Proof: We only consider the case $\varepsilon<1$. In the previous lemma we choose $n=0$ and $\zeta$ such that $\mu(\zeta)=\lambda_{0}$, which is possible since $\Theta_{0}<\lambda_{0}<1$ and $\mu(\zeta)$ takes all values of $\left[\Theta_{0}, 1\right)$ when $\zeta$ lays in $\mathbb{R}_{+}$. Thus we get (3.12).

In the next section, we improve the estimate of Theorem 1.5 for each fixed rank $n$ when $\theta$ goes to zero.

\section{Asymptotics of eigenvalues in the small angle limit}

As it has been proved in Theorem 1.5, when $\theta$ goes to zero, the number of eigenvalues $n(\theta)$ below the essential spectrum tends to infinity. Thus, for any arbitrary integer $N$, we can find a value of $\theta$ small enough such that $\sigma_{N}(\theta)<1$. In this section, we investigate the asymptotics of those eigenvalues and prove Theorem 1.7. We use again the scaling (3.3):

$$
\left\{\begin{array}{l}
y=s \sqrt{\sin \theta}-\frac{\zeta_{0}}{\sqrt{\tan \theta}} \\
z=t \sqrt{\cos \theta}
\end{array}\right.
$$

In the new variables, the operator $\mathcal{L}_{\theta}$ rewrites

$$
\sin \theta D_{y}^{2}+\cos \theta D_{z}^{2}+\cos \theta\left(z-\zeta_{0}-y \sqrt{\tan \theta}\right)^{2}=\cos \theta\left(\mathfrak{L}_{h}+\Theta_{0}\right),
$$

where we have set $h=\tan \theta$ and

$$
\mathfrak{L}_{h}=h D_{y}^{2}+D_{z}^{2}+\left(z-\zeta_{0}-y h^{1 / 2}\right)^{2}-\Theta_{0}
$$


We denote by $\mathfrak{s}_{n}(h)$ the $n$-th eigenvalue of $\mathfrak{L}_{h}$. Due to the change of variables, we have

$$
\sigma_{n}(\theta)=\cos \theta\left(\Theta_{0}+\mathfrak{s}_{n}(\tan \theta)\right) \text {. }
$$

Thus, Theorem 1.7 is clearly a consequence of the following asymptotics for $\mathfrak{s}_{n}(h)$ which we are going to establish:

Theorem 4.1 For all $n \geq 1$, there exists a sequence $\left(b_{j, n}\right)_{j \geq 0}$ such that for all $N \geq 1$ and $J \geq 1$, there exist $C_{N, J}>0$ and $h_{0}>0$ such that for all $1 \leq n \leq N$ and $0<h<h_{0}$ :

$$
\left|\mathfrak{s}_{n}(h)-\sum_{j=0}^{J} b_{j, n} h^{j}\right| \leq C_{N, J} h^{J+1} .
$$

Moreover $b_{0, n}=0$ and $b_{1, n}=(2 n-1) \sqrt{\frac{\mu^{\prime \prime}\left(\zeta_{0}\right)}{2}}$.

Remark 4.2 It follows from Theorem 4.1 that for $h$ small enough the eigenvalues $\mathfrak{s}_{n}(h)$, $1 \leq n \leq N$, are simple.

The proof of Theorem 4.1 is organized in two main steps. Using the one-dimensional operators $H_{\zeta}$ defined in (1.2), we can rewrite (4.1) as

$$
\mathfrak{L}_{h}=h D_{y}^{2}+H_{\zeta_{0}+y \sqrt{h}}\left(z ; D_{z}\right)-\Theta_{0}
$$

In a first step we construct quasimodes by an expansion in powers of $h^{1 / 2}$ (natural power of $h$ appearing in $\mathfrak{L}_{h}$ ), and using the spectral theorem, we get a family of approximate eigenvalues (constructed as asymptotic series in powers of $h^{1 / 2}$ and whose odd terms will be zero for some parity reason) and a rough upper bound for $\mathfrak{s}_{n}(h)$. In a second step, we establish a lower bound. The basic idea to get such a lower bound is to use a BornOppenheimer technique which consists of replacing $H_{\zeta_{0}+y \sqrt{h}}$ by its ground energy $\mu\left(\zeta_{0}+\right.$ $y \sqrt{h}$ ) and to implement the standard harmonic approximation in the semi-classical limit for the one-dimensional operator $\mathfrak{L}_{h, \mathrm{BO}}$ defined as:

$$
\mathfrak{L}_{h, \mathrm{BO}}=h D_{y}^{2}+\mu\left(\zeta_{0}+y \sqrt{h}\right)-\Theta_{0}
$$

However $\mathfrak{L}_{h, \text { BO }}$, seen as an operator acting on the domain of $\mathfrak{L}_{h}-$ i.e. as two-dimensional operator, has eigenvalues of infinite multiplicity, and we cannot use directly the min-max principle to compare its spectrum with the eigenvalues of $\mathfrak{L}_{h}$. Thus, we have to justify, through Agmon estimates and a Grushin type argument, that the eigenvalues of $\mathfrak{L}_{h}$ are bounded from below by those of $\mathfrak{L}_{h, \mathrm{BO}}$ seen as one-dimensional operator. Such a procedure was described in [17] for degenerate potentials in $\mathbb{R}^{n}$. Nevertheless, we cannot apply directly the techniques of [17] because the minimal line of the potential $V_{\theta}$ goes to infinity and we work in a domain with boundary. 


\subsection{Construction of quasimodes}

We can write $\mathfrak{L}_{h}$ as:

$$
\mathfrak{L}_{h}=P_{0}+h^{1 / 2} P_{1}+h P_{2}
$$

with:

$$
\begin{aligned}
& P_{0}=D_{z}^{2}+\left(z-\zeta_{0}\right)^{2}-\Theta_{0}=H_{\zeta_{0}}-\Theta_{0}, \\
& P_{1}=-2\left(z-\zeta_{0}\right) y, \\
& P_{2}=D_{y}^{2}+y^{2} .
\end{aligned}
$$

We look for formal series solution of the equation $\mathfrak{L}_{h} u_{h}=\gamma_{h} u_{h}$ in the form:

$$
u_{h} \simeq \sum_{j \geq 0} \varphi_{j} h^{j / 2} \quad \text { and } \quad \gamma_{h}=\sum_{j \geq 0} \gamma_{j} h^{j / 2}
$$

We are led to the system:

$$
\begin{aligned}
h^{0}: & \left(P_{0}-\gamma_{0}\right) \varphi_{0}=0, \\
h^{1 / 2}: & \left(P_{0}-\gamma_{0}\right) \varphi_{1}=\gamma_{1} \varphi_{0}-P_{1} \varphi_{0}, \\
h: & \left(P_{0}-\gamma_{0}\right) \varphi_{2}=\gamma_{2} \varphi_{0}+\gamma_{1} \varphi_{1}-P_{2} \varphi_{0}-P_{1} \varphi_{1}, \\
h^{j / 2}: & \left(P_{0}-\gamma_{0}\right) \varphi_{j}=\sum_{k=0}^{j-1} \gamma_{j-k} \varphi_{k}-P_{2} \varphi_{j-2}-P_{1} \varphi_{j-1} .
\end{aligned}
$$

Order $h^{0}$. Considering (4.2), we choose $\gamma_{0}=0$ and the general solution of (4.5) is

$$
\varphi_{0}(y, z)=f_{0}(y) v_{\zeta_{0}}(z)
$$

for some $f_{0}$ to determine.

Order $h^{1 / 2}$. To solve (4.6), a necessary and sufficient compatibility condition is:

$$
\left\langle\gamma_{1} \varphi_{0}(y, \cdot)-P_{1} \varphi_{0}(y, \cdot), v_{\zeta_{0}}\right\rangle_{z}=0, \quad \forall y \in \mathbb{R}
$$

where $\langle\cdot, \cdot\rangle_{z}$ denotes the standard $L^{2}$ scalar product on $\mathbb{R}_{+}$with respect to $z$. Using (4.3) and (4.9) this condition becomes

$$
\gamma_{1} f_{0}(y)+2 y f_{0}(y)\left\langle\left(z-\zeta_{0}\right) v_{\zeta_{0}}, v_{\zeta_{0}}\right\rangle_{z}=0, \quad \forall y \in \mathbb{R} .
$$

In order to evaluate the scalar product, we recall an easy computation. Let us take the derivative with respect to $\zeta$ of:

$$
\left(H_{\zeta}-\mu(\zeta)\right) v_{\zeta}=0
$$


Choosing $\zeta=\zeta_{0}$, we get:

$$
\left.\left(H_{\zeta_{0}}-\Theta_{0}\right)\left(\partial_{\zeta} v_{\zeta}\right)\right|_{\zeta=\zeta_{0}}=2\left(z-\zeta_{0}\right) v_{\zeta_{0}}
$$

We deduce:

$$
\int_{\mathbb{R}_{+}}\left(z-\zeta_{0}\right) v_{\zeta_{0}}^{2}(z) \mathrm{d} z=0 .
$$

By (4.11), we get $\gamma_{1}=0$ and, thanks to (4.10), the general solution of (4.6) is given by:

$$
\varphi_{1}(y, z)=y f_{0}(y) w_{\zeta_{0}}(z)+f_{1}(y) v_{\zeta_{0}}(z), \quad \text { with } \quad w_{\zeta_{0}}(z):=\left.\left(\partial_{\zeta} v_{\zeta}\right)\right|_{\zeta=\zeta_{0}},
$$

and where $f_{1}$ is to be determined.

Order $h$. Taking (4.9) and (4.12) into account we rewrite equation (4.7) in the form

$$
\left(H_{\zeta_{0}}-\Theta_{0}\right) \varphi_{2}=\gamma_{2} f_{0}(y) v_{\zeta_{0}}(z)-P_{2} f_{0}(y) v_{\zeta_{0}}(z)-P_{1} f_{1}(y) v_{\zeta_{0}}(z)-P_{1} y f_{0}(y) w_{\zeta_{0}}(z)
$$

From the previous calculations, we already know that a particular solution of the equation $\left(H_{\zeta_{0}}-\Theta_{0}\right) \varphi=-P_{1} f_{1}(y) v_{\zeta_{0}}(z)$ is $y f_{1}(y) w_{\zeta_{0}}(z)$. That is why we look for $\varphi_{2}$ in the general form

$$
\varphi_{2}(y, z)=\varphi_{2}^{\perp}(y, z)+y f_{1}(y) w_{\zeta_{0}}(z)+f_{2}(y) v_{\zeta_{0}}(z)
$$

where $\left\langle\varphi_{2}^{\perp}(y, z), v_{\zeta_{0}}\right\rangle_{z}=0$ for all $y \in \mathbb{R}$. Note that as a consequence of the equality $\left\langle v_{\zeta}, v_{\zeta}\right\rangle_{z}=1$ for all $\zeta$, we have

$$
\left\langle w_{\zeta_{0}}, v_{\zeta_{0}}\right\rangle_{z}=0
$$

Thus $\varphi_{2}^{\perp}$ has to solve

$$
\left(H_{\zeta_{0}}-\Theta_{0}\right) \varphi_{2}^{\perp}=\gamma_{2} f_{0}(y) v_{\zeta_{0}}(z)-P_{2} f_{0}(y) v_{\zeta_{0}}(z)-P_{1} y f_{0}(y) w_{\zeta_{0}}(z) .
$$

The corresponding compatibility condition is:

$$
\left\langle\gamma_{2} f_{0}(y) v_{\zeta_{0}}-P_{2} f_{0}(y) v_{\zeta_{0}}-P_{1} y f_{0}(y) w_{\zeta_{0}}, v_{\zeta_{0}}\right\rangle_{z}=0, \quad \forall y \in \mathbb{R}
$$

i.e.

$$
\gamma_{2} f_{0}(y)=P_{2} f_{0}(y)-2 y^{2} f_{0}(y)\left\langle\left(z-\zeta_{0}\right) w_{\zeta_{0}}, v_{\zeta_{0}}\right\rangle_{z}, \quad \forall y \in \mathbb{R}
$$

But we have the identity:

$$
\left(H_{\zeta_{0}}-\Theta_{0}\right) v_{\zeta_{0}}^{(2)}=\left(\mu^{\prime \prime}\left(\zeta_{0}\right)-2\right) v_{\zeta_{0}}+4\left(z-\zeta_{0}\right) w_{\zeta_{0}} \quad \text { with } \quad v_{\zeta_{0}}^{(2)}(z):=\left.\left(\partial_{\zeta}^{2} v_{\zeta}\right)\right|_{\zeta=\zeta_{0}} .
$$

Taking the scalar product $\left\langle\bullet, v_{\zeta_{0}}\right\rangle_{z}$, we find the well-known identity (see [3, p. 12831284] and also [9]):

$$
\mu^{\prime \prime}\left(\zeta_{0}\right)-2=-4\left\langle\left(z-\zeta_{0}\right) w_{\zeta_{0}}, v_{\zeta_{0}}\right\rangle_{z}
$$


and the compatibility condition (4.14) becomes

$$
H_{\text {harm }} f_{0}=\gamma_{2} f_{0}, \quad \text { with } \quad H_{\text {harm }}=D_{y}^{2}+\frac{\mu^{\prime \prime}\left(\zeta_{0}\right)}{2} y^{2} .
$$

Thus, for $f_{0}$ we take an eigenfunction $f_{n \text {, harm }}$ of $H_{\text {harm }}$, and for $\gamma_{2}$ the associated eigenvalue

$$
\gamma_{2}=\lambda_{n, \text { harm }}:=\sqrt{\frac{\mu^{\prime \prime}\left(\zeta_{0}\right)}{2}}(2 n-1) \quad(n \geq 1) .
$$

With this choice, $\varphi_{2}^{\perp}$ exists and is unique.

Further terms Let us assume that the coefficients $\left(\gamma_{k}\right)_{0 \leq k \leq j}$ are determined. Let us also assume that, for $0 \leq k \leq j$ the functions $\varphi_{k}$ can be written in the form:

$$
\varphi_{k}(y, z)=\varphi_{k}^{\perp}(y, z)+y f_{k-1}(y) w_{\zeta_{0}}(z)+f_{k}(y) v_{\zeta_{0}}(z)
$$

where $\left\langle\varphi_{k}^{\perp}(y, \cdot), v_{\zeta_{0}}\right\rangle_{z}=0$ for all $y \in \mathbb{R}$ and with the convention $f_{-1}=0$. We assume that $\left(\varphi_{k}^{\perp}\right)_{0 \leq k \leq j}$ and $\left(f_{k}\right)_{0 \leq k \leq j-2}$ determined in $\mathcal{S}\left(\mathbb{R} \times \mathbb{R}_{+}\right)$and $\mathcal{S}(\mathbb{R})$, respectively, $f_{j-1}$ and $f_{j}$ being still unknown.

This assumption is proven for $j \leq 2$. Let us prove it for $j+1$. For this, we write the equation of order $j+1$ :

$$
\left(H_{\zeta_{0}}-\Theta_{0}\right) \varphi_{j+1}=\sum_{k=0}^{j} \gamma_{j+1-k} \varphi_{k}-P_{2} \varphi_{j-1}-P_{1} \varphi_{j}
$$

We write $\varphi_{j+1}$ in the form:

$$
\varphi_{j+1}(y, z)=\varphi_{j+1}^{\perp}(y, z)+y f_{j}(y) w_{\zeta_{0}}(z)+f_{j+1}(y) v_{\zeta_{0}}(z) .
$$

Then equation (4.18) implies the following equation in $\varphi_{j+1}^{\perp}$

$$
\left(H_{\zeta_{0}}-\Theta_{0}\right) \varphi_{j+1}^{\perp}=\gamma_{j+1} f_{0} v_{\zeta_{0}}+\gamma_{2} f_{j-1} v_{\zeta_{0}}-P_{2}\left(f_{j-1} v_{\zeta_{0}}\right)-P_{1}\left(y f_{j-1} w_{\zeta_{0}}\right)+R_{j}
$$

where

$$
R_{j}=\sum_{k=1}^{j-2} \gamma_{j+1-k} \varphi_{k}+\gamma_{2} \varphi_{j-1}^{\perp}+\gamma_{2} y f_{j-2} w_{\zeta_{0}}-P_{2} \varphi_{j-1}^{\perp}-P_{2}\left(y f_{j-2} w_{\zeta_{0}}\right)-P_{1} \varphi_{j}^{\perp}
$$

is known and belongs to $\mathcal{S}\left(\mathbb{R} \times \mathbb{R}_{+}\right)$. The compatibility condition ensuring the solvability of (4.19) is obtained by taking the scalar product with $v_{\zeta_{0}}$. We calculate, $\mathrm{cf}(4.14)$

$\gamma_{j+1} f_{0}(y)+\gamma_{2} f_{j-1}(y)=\left(P_{2} f_{j-1}\right)(y)-2 y^{2} f_{j-1}(y)\left\langle\left(z-\zeta_{0}\right) w_{\zeta_{0}}, v_{\zeta_{0}}\right\rangle_{z}-g_{j}(y), \quad \forall y \in \mathbb{R}$ 
where $g_{j}=\left\langle R_{j}, v_{\zeta_{0}}\right\rangle_{z}$ belongs to $\mathcal{S}(\mathbb{R})$. Thanks to (4.15) this equation in $y$ can be put in the form:

$$
\left(H_{\text {harm }}-\gamma_{2}\right) f_{j-1}=\gamma_{j+1} f_{0}+g_{j} .
$$

The compatibility condition ensuring the solvability of (4.20) is obtained by taking the scalar product with $f_{0}=f_{n \text {, harm: }}$ :

$$
\gamma_{j+1}+\left\langle g_{j}, f_{0}\right\rangle_{y}=0
$$

This determines $\gamma_{j+1}$, and then $f_{j-1}$ (the unique solution orthogonal to $f_{0}$ ) thanks to the Fredholm alternative. The assertion is proven at the order $j+1$.

Cancellation of odd terms Let us now explain why, for $j$ odd, we have $\gamma_{j}=0$. Let us first notice that either $f_{0}$ is odd or $f_{0}$ is even and that $P_{1}$ is odd and $P_{2}$ is even (with respect to $y$ ) and also that $\gamma_{1}=0$. To fix ideas, we deal with the case $f_{0}$ even, the other one being completely similar. Then, we observe that $\varphi_{1}^{\perp}$ is odd with respect to $y$ and $\varphi_{2}^{\perp}$ is even.

In the recursion above, we can assume that, for $0 \leq k \leq j, \varphi_{k}^{\perp}$ is even/odd if $k$ is even/odd (with respect to $y$ ) and that the already known $f_{k}$ are even/odd if $k$ is even/odd. In addition, we assume that $\gamma_{k}=0$ if $k$ is odd and $k \leq j$. Using this recursion assumption, we get that, if $j$ is even/odd, then $R_{j}$ (and thus $g_{j}$ ) is odd/even.

If $j$ is even, we get $\left\langle g_{j}, f_{0}\right\rangle=0$, thus $\gamma_{j+1}=0$. Then the Fredholm condition (see (4.20)) implies that $f_{j-1}$ is odd. Coming back to (4.19), we check that the other terms in the right hand side are odd with respect to $y$. We have that $\varphi_{j+1}^{\perp}$ is orthogonal to $v_{\zeta_{0}}$, thus we deduce by uniqueness that $\varphi_{j+1}^{\perp}$ is odd. If $j$ is odd, then $\gamma_{j+1}$ does not need to be zero and $f_{j-1}$ is even. We deduce in the same way that $\varphi_{j+1}^{\perp}$ is even. Thus the assertion is proved by recursion.

This analysis provides a quasimode for $\mathfrak{L}_{h}($ for all $n$ and $J)$ :

$$
\mathfrak{u}_{n}^{J}(h)=\sum_{j=0}^{J} \varphi_{j} h^{j / 2}
$$

which satisfies

$$
\left\|\left(\mathfrak{L}_{h}-\sum_{j=0}^{J} \gamma_{2 j, n} h^{j}\right) \mathfrak{u}_{n}^{J}(h)\right\|_{L^{2}(\Omega)} \leq C_{n, J} h^{J+1}\left\|\mathfrak{u}_{n}^{J}(h)\right\|_{L^{2}(\Omega)},
$$

where we use the notation $\gamma_{2 j, n}$ for $\gamma_{2 j}$ to emphasize the dependence on $n$. Using the spectral theorem, we immediately deduce that:

Proposition 4.3 For all $N \geq 1$ and $J \geq 1$, there exist $C_{N, J}>0$ and $h_{0}>0$ such that for all $1 \leq n \leq N$ and $0<h<h_{0}$ :

$$
\operatorname{dist}\left(\operatorname{sp}_{\operatorname{dis}}\left(\mathfrak{L}_{h}\right), \sum_{j=0}^{J} \gamma_{2 j, n} h^{j}\right) \leq C_{N, J} h^{J+1} .
$$


Remark 4.4 In particular, we observe that, for $1 \leq n \leq N$ and $h \in\left(0, h_{0}\right)$ :

$$
0 \leq \mathfrak{s}_{n}(h) \leq h \lambda_{n, \text { harm }}+C_{N, 1} h^{2} \leq C_{N} h .
$$

\subsection{Lower bound}

To get a suitable lower bound of $\mathfrak{s}_{n}(h)$, we will use the so-called Born-Oppenheimer approximation $\mathfrak{L}_{h, \mathrm{BO}}$ with

$$
\mathfrak{L}_{h, \mathrm{BO}}:=h D_{y}^{2}+W_{h}(y), \quad \text { with } \quad W_{h}(y)=\mu\left(\zeta_{0}+y h^{1 / 2}\right)-\Theta_{0} \geq 0 .
$$

Thus, we have

$$
\forall v \in D_{N}\left(\mathfrak{L}_{h}\right), \quad\left\langle\mathfrak{L}_{h} v, v\right\rangle \geq\left\langle\mathfrak{L}_{h, \mathrm{BO}} v, v\right\rangle .
$$

\subsubsection{Localization estimates of Agmon type}

Let us take $N_{0}$ such that $1 \leq N_{0} \leq N$. We are going to prove some localization of the eigenfunctions of $\mathfrak{L}_{h}$ associated with $\left(\mathfrak{s}_{n}(h)\right)_{1 \leq n \leq N_{0}}$. For all $1 \leq n \leq N_{0}$, we will consider a normalized eigenfunction $\mathfrak{u}_{n}(h)$ associated with $\mathfrak{s}_{n}(h)$ so that the distinct $\mathfrak{u}_{n}(h)$ are orthogonal. It is convenient to introduce the sum of the first eigenspaces of $\mathfrak{L}_{h}$ :

$$
\mathfrak{E}_{N_{0}}(h)=\operatorname{span}\left(\mathfrak{u}_{1}(h), \ldots, \mathfrak{u}_{N_{0}}(h)\right) .
$$

Combining Proposition 2.6 and the scaling (3.3), we have the following localization with respect to the normal variable $z$ :

Proposition 4.5 There exist $C>0, \gamma>0$ and $h_{0}>0$ such that for all $h \in\left(0, h_{0}\right)$ and $v \in \mathfrak{E}_{N_{0}}(h)$ :

$$
\int_{\Omega} e^{2 \gamma z}|v|^{2} \mathrm{~d} y \mathrm{~d} z \leq C\|v\|_{L^{2}(\Omega)}^{2} .
$$

Now we improve Theorem 1.3 by proving an optimal localization with respect to $y$ when $h$ goes to 0 :

Proposition 4.6 There exist $C>0$ and $h_{0}>0$ such that for all $h \in\left(0, h_{0}\right)$ and $v \in$ $\mathfrak{E}_{N_{0}}(h)$ :

$$
\int_{\Omega} e^{2|y|}|v|^{2} \mathrm{~d} y \mathrm{~d} z \leq C\|v\|_{L^{2}(\Omega)}^{2}
$$

Proof: For $v=\mathfrak{u}_{n}(h)$, we can write:

$$
\mathfrak{q}_{h}\left(e^{\Phi} v\right)-\int_{\Omega}\left(\left|D_{z} \Phi\right|^{2}+h\left|D_{y} \Phi\right|^{2}+\mathfrak{s}_{n}(h)\right)\left|e^{\Phi} v\right|^{2} \mathrm{~d} y \mathrm{~d} z=0,
$$


where $\mathfrak{q}_{h}$ is the quadratic form associated to (4.1). Let us choose $\Phi(y)=|y|$. With (4.23), we deduce:

$$
\int_{\Omega}\left(W_{h}-h-\mathfrak{s}_{n}(h)\right)\left|e^{\Phi} v\right|^{2} \mathrm{~d} y \mathrm{~d} z \leq 0
$$

Let us consider the positivity of:

$$
W_{h}-h-\mathfrak{s}_{n}(h)
$$

in a region of the type $|y| \geq C_{0}$ with $C_{0}>0$.

(i) Using the non-degeneracy of the minimum, we know that it exists $\varepsilon_{0}$ such that:

$$
W_{h}(y) \geq \frac{\mu^{\prime \prime}\left(\zeta_{0}\right)}{4}|y|^{2} h, \quad \text { for }|y| \leq \varepsilon_{0} h^{-1 / 2} .
$$

(ii) With $\eta_{0}:=\min \left\{\mu\left(\zeta_{0} \pm \varepsilon_{0}\right)\right\}-\Theta_{0}$, we have $W_{h}(y) \geq \eta_{0}>0$ for $|y| \geq \varepsilon_{0} h^{-1 / 2}$. We deduce from $(i)$ and (ii) that

$$
W_{h}(y) \geq \min \left\{\eta_{0}, \frac{\mu^{\prime \prime}\left(\zeta_{0}\right)}{4} C_{0}^{2} h\right\} \quad \text { if } \quad|y| \geq C_{0}
$$

Therefore, using Remark 4.4 and choosing $C_{0}$ large enough, and $h$ small enough we get the existence of $c>0$ such that, for $|y| \geq C_{0}$ :

$$
W_{h}(y)-h-\mathfrak{s}_{n}(h) \geq c h .
$$

Combining this with (4.28), we obtain:

$$
\operatorname{ch} \int_{|y| \geq C_{0}}\left|e^{\Phi} v\right|^{2} \mathrm{~d} y \mathrm{~d} z \leq \int_{|y| \leq C_{0}}\left|W_{h}-h-\mathfrak{s}_{n}(h)\right|\left|e^{\Phi} v\right|^{2} \mathrm{~d} y \mathrm{~d} z .
$$

Then we take advantage of Remark 4.4 and bound $W_{h}(y)$ for $|y| \leq C_{0}$ by $C_{0}^{\prime} h$ for a suitable constant $C_{0}^{\prime}$ to deduce finally

$$
\operatorname{ch} \int_{|y| \geq C_{0}}\left|e^{\Phi} v\right|^{2} \mathrm{~d} y \mathrm{~d} z \leq C h\|v\|_{L^{2}(\Omega)}^{2} .
$$

Thus we have proved (4.26) for $v=\mathfrak{u}_{n}(h), 1 \leq n \leq N_{0}$. Using the orthogonality of the eigenvectors $\mathfrak{u}_{n}(h)$, we obtain (4.26) for $v \in \mathfrak{E}_{N_{0}}(h)$.

Combining Propositions 4.5 and 4.6, we get the following two corollaries:

Corollary 4.7 There exist $C>0, \delta>0$ and $h_{0}>0$ such that for all $h \in\left(0, h_{0}\right)$ and $v \in \mathfrak{E}_{N_{0}}(h)$ :

$$
\int_{\Omega} e^{\delta(|y|+z)}|v|^{2} \mathrm{~d} y \mathrm{~d} z \leq C\|v\|_{L^{2}(\Omega)}^{2},
$$

and in particular:

$$
\int_{\Omega}\left(1+z|y|^{3}+y^{6}\right)|v|^{2} \mathrm{~d} y \mathrm{~d} z \leq C\|v\|_{L^{2}(\Omega)}^{2} .
$$


Corollary 4.8 For all $\varepsilon_{0}>0$, there exist $h_{0}>0, \gamma>0$ and $C>0$ such that, for all $h \in\left(0, h_{0}\right)$ and $v \in \mathfrak{E}_{N_{0}}(h)$ :

$$
\int_{|y| \geq \varepsilon_{0} h^{-1 / 2}}\left(1+|y|^{4}\right)|v|^{2} \mathrm{~d} y \mathrm{~d} z \leq C e^{-\gamma h^{-1 / 2}}\|v\|_{L^{2}(\Omega)}^{2} .
$$

\subsubsection{Approximation of eigenvectors}

We will consider the projection $\Pi_{0}: L^{2}(\Omega) \rightarrow L^{2}(\mathbb{R}) \otimes \operatorname{span}\left\{v_{\zeta_{0}}\right\}$ defined by

$$
w \longmapsto\left\langle w, v_{\zeta_{0}}\right\rangle_{z} v_{\zeta_{0}}
$$

The aim of the following proposition is to approximate $\mathfrak{u}_{n}(h)$ by a tensor product:

Proposition 4.9 There exist $C>0$ and $h_{0}>0$ such that for all $h \in\left(0, h_{0}\right)$ :

$$
\begin{aligned}
Q\left(\mathfrak{u}_{n}(h)-\Pi_{0} \mathfrak{u}_{n}(h)\right) & \leq C h^{1 / 2}\left\|\mathfrak{u}_{n}(h)\right\|_{L^{2}(\Omega)}^{2}, \\
Q\left(D_{y} \mathfrak{u}_{n}(h)-\Pi_{0} D_{y} \mathfrak{u}_{n}(h)\right) & \leq C h^{1 / 4}\left\|\mathfrak{u}_{n}(h)\right\|_{L^{2}(\Omega)}^{2}, \\
Q\left(y \mathbf{u}_{n}(h)-\Pi_{0} y \mathbf{u}_{n}(h)\right) & \leq C h^{1 / 2}\left\|\mathbf{u}_{n}(h)\right\|_{L^{2}(\Omega)}^{2},
\end{aligned}
$$

where $Q$ is the quadratic form of $I d_{L^{2}(\mathbb{R})} \otimes\left(H_{\zeta_{0}}-\Theta_{0}\right)$.

Proof: 1) We first notice that, since $\Theta_{0}$ is the first eigenvalue of $H_{\zeta_{0}}$, the quadratic form $Q$ is non-negative: $Q(w) \geq 0$ for all $w \in L^{2}(\mathbb{R}) \otimes B^{1}\left(\mathbb{R}_{+}\right)$.

2) We also notice that, since $v_{\zeta_{0}}$ generates the kernel of $H_{\zeta_{0}}-\Theta_{0}$, there holds

$$
Q(w)=Q\left(w-\Pi_{0} w\right), \quad \forall w \in L^{2}(\mathbb{R}) \otimes B^{1}\left(\mathbb{R}_{+}\right) .
$$

Hence we only have to bound $Q\left(\mathfrak{u}_{n}(h)\right), Q\left(D_{y} \mathfrak{u}_{n}(h)\right)$, and $Q\left(y \mathfrak{u}_{n}(h)\right)$.

3) Using the equation satisfied by $\mathfrak{u}_{n}(h)$ :

$$
\mathfrak{L}_{h} \mathfrak{u}_{n}(h)=\mathfrak{s}_{n}(h) \mathfrak{u}_{n}(h),
$$

and taking the scalar product with $\mathfrak{u}_{n}(h)$, we find the identity

$$
\begin{aligned}
h \| D_{y} \mathbf{u}_{n}(h) & \|_{L^{2}(\Omega)}^{2}+Q\left(\mathbf{u}_{n}(h)\right) \\
& -2 h^{1 / 2}\left\langle\left(z-\zeta_{0}\right) y \mathbf{u}_{n}(h), \mathbf{u}_{n}(h)\right\rangle+h\left\|y \mathbf{u}_{n}(h)\right\|_{L^{2}(\Omega)}^{2}=\mathfrak{s}_{n}(h)\left\|\mathbf{u}_{n}(h)\right\|_{L^{2}(\Omega)}^{2}
\end{aligned}
$$

With (4.22) and (4.31), we deduce

$$
Q\left(\mathfrak{u}_{n}(h)\right) \leq C h^{1 / 2}\left\|\mathbf{u}_{n}(h)\right\|_{L^{2}(\Omega)}^{2} .
$$

4) Considering again the scalar product of identity (4.32) with $\mathfrak{u}_{n}(h)$, we observe that, using (4.22) and (4.23):

$$
\left\|D_{y} \mathfrak{u}_{n}(h)\right\|_{L^{2}(\Omega)}^{2} \leq C\left\|\mathbf{u}_{n}(h)\right\|_{L^{2}(\Omega)}^{2} .
$$


Moreover, calculating the derivative with respect to $y$ of (4.32), we obtain:

$$
\mathfrak{L}_{h} D_{y} \mathfrak{u}_{n}(h)+2 i h^{1 / 2}\left(z-\zeta_{0}-y h^{1 / 2}\right) \mathbf{u}_{n}(h)=\mathfrak{s}_{n}(h) D_{y} \mathfrak{u}_{n}(h) .
$$

Taking the scalar product with $D_{y} \mathfrak{u}_{n}(h)$, we get:

$$
\left\|D_{y}^{2} \mathfrak{u}_{n}(h)\right\|_{L^{2}(\Omega)}^{2} \leq C h^{-1 / 2}\left\|\mathbf{u}_{n}(h)\right\|_{L^{2}(\Omega)}^{2},
$$

where we have used (4.31) to control the commutator term.

Considering once more the scalar product of (4.34) with $D_{y} \mathfrak{u}_{n}(h)$, we infer:

$$
\begin{aligned}
& h\left\|D_{y}^{2} \mathfrak{u}_{n}(h)\right\|_{L^{2}(\Omega)}^{2}+Q\left(D_{y} \mathbf{u}_{n}(h)\right)+2 i h^{1 / 2}\left\langle\left(z-\zeta_{0}-y h^{1 / 2}\right) \mathfrak{u}_{n}(h), D_{y} \mathfrak{u}_{n}(h)\right\rangle \\
- & 2 h^{1 / 2}\left\langle\left(z-\zeta_{0}\right) y D_{y} \mathfrak{u}_{n}(h), D_{y} \mathfrak{u}_{n}(h)\right\rangle+h\left\|y D_{y} \mathfrak{u}_{n}(h)\right\|_{L^{2}(\Omega)}^{2}=\mathfrak{s}_{n}(h)\left\|D_{y} \mathfrak{u}_{n}(h)\right\|_{L^{2}(\Omega)}^{2}
\end{aligned}
$$

With the help of (4.31), (4.33), (4.35) and integration by parts, we find

$$
Q\left(D_{y} \mathfrak{u}_{n}(h)\right) \leq C h^{1 / 4}\left\|\mathbf{u}_{n}(h)\right\|_{L^{2}(\Omega)}^{2} .
$$

5) Similarly, we multiply (4.32) by $y$ and find the identity

$$
\mathfrak{L}_{h}\left(y \mathbf{u}_{n}(h)\right)+2 h \partial_{y} \mathfrak{u}_{n}(h)=\mathfrak{s}_{n}(h) y \mathfrak{u}_{n}(h),
$$

from which we deduce

$$
\begin{aligned}
& h\left\|D_{y}\left(y \mathfrak{u}_{n}(h)\right)\right\|_{L^{2}(\Omega)}^{2}+Q\left(y \mathfrak{u}_{n}(h)\right)+2 h\left\langle\partial_{y} \mathfrak{u}_{n}(h), y \mathfrak{u}_{n}(h)\right\rangle \\
& \quad-2 h^{1 / 2}\left\langle\left(z-\zeta_{0}\right) y^{2} \mathfrak{u}_{n}(h), y \mathfrak{u}_{n}(h)\right\rangle+h\left\|y^{2} \mathfrak{u}_{n}(h)\right\|_{L^{2}(\Omega)}^{2}=\mathfrak{s}_{n}(h)\left\|y \mathfrak{u}_{n}(h)\right\|_{L^{2}(\Omega)}^{2} .
\end{aligned}
$$

We obtain finally

$$
Q\left(y \mathfrak{u}_{n}(h)\right) \leq C h^{1 / 2}\left\|\mathfrak{u}_{n}(h)\right\|_{L^{2}(\Omega)}^{2},
$$

which concludes the proof.

Corollary 4.10 There exist $C>0$ and $h_{0}>0$ such that for all $h \in\left(0, h_{0}\right)$ and $v \in$ $\mathfrak{E}_{N_{0}}(h)$ :

$$
\left\|v-\Pi_{0} v\right\|_{H^{1}(\Omega)}+\left\|y v-\Pi_{0} y v\right\|_{L^{2}(\Omega)} \leq C h^{1 / 8}\|v\|_{L^{2}(\Omega)} .
$$

Proof: Let us assume that $w \in L^{2}(\mathbb{R}) \otimes B^{1}\left(\mathbb{R}_{+}\right)$is such that: $\left\langle w, v_{\zeta_{0}}\right\rangle_{L^{2}\left(\mathbb{R}_{+}, z\right.}=0$. Then, we get:

$$
Q(w) \geq\left(\mu_{2}\left(\zeta_{0}\right)-\Theta_{0}\right)\|w\|_{L^{2}(\Omega)}^{2},
$$

where $\mu_{2}\left(\zeta_{0}\right)$ denotes the second eigenvalue of $H_{\zeta_{0}}$. Therefore the left-hand side of (4.37) is bounded by $\left(\mu_{2}\left(\zeta_{0}\right)-\Theta_{0}\right)^{-1}\left\{Q(w)+Q\left(D_{y} w\right)+Q(y w)\right\}$. Then, the conclusion follows from Proposition 4.9 .

Corollary 4.11 There exists $h_{0}>0$ such that for $h \in\left(0, h_{0}\right)$, the projection $\Pi_{0}$ is an isomorphism from $\mathfrak{E}_{N_{0}}(h)$ onto its range. 


\subsubsection{Conclusion}

For all $v \in \mathfrak{E}_{N_{0}}(h)$, we have

$$
\left\langle\left(h D_{y}^{2}+\mu\left(\zeta_{0}+y h^{1 / 2}\right)-\Theta_{0}\right) v, v\right\rangle \leq \mathfrak{s}_{N_{0}}(h)\|v\|_{L^{2}(\Omega)}^{2} .
$$

We recall (1.3) and we have, with Corollary 4.8:

$$
\int_{|y| \geq \varepsilon_{0} h^{-1 / 2}}\left|\left(\mu\left(y h^{1 / 2}+\zeta_{0}\right)-\Theta_{0}-h \frac{\mu^{\prime \prime}\left(\zeta_{0}\right)}{2} y^{2}\right) v\right|^{2} \mathrm{~d} y \mathrm{~d} z=\mathcal{O}\left(h^{\infty}\right)\|v\|_{L^{2}(\Omega)}^{2} .
$$

A Taylor approximation (using that $\mu$ is smooth) gives:

$$
\begin{aligned}
\int_{|y| \leq \varepsilon_{0} h^{-1 / 2}}\left|\left(\mu\left(y h^{1 / 2}+\zeta_{0}\right)-\Theta_{0}-h \frac{\mu^{\prime \prime}\left(\zeta_{0}\right)}{2} y^{2}\right) v\right|^{2} \mathrm{~d} y \mathrm{~d} z & \leq C\left(\varepsilon_{0}\right)\left\|y^{3} h^{3 / 2} v\right\|_{L^{2}(\Omega)}^{2} \\
& \leq \tilde{C}\left(\varepsilon_{0}\right) h^{3}\|v\|_{L^{2}(\Omega)}^{2}
\end{aligned}
$$

the last inequality coming from (4.31). We get:

$$
h\left\langle\left(D_{y}^{2}+\frac{\mu^{\prime \prime}\left(\zeta_{0}\right)}{2} y^{2}\right) v, v\right\rangle-C h^{3 / 2}\|v\|_{L^{2}(\Omega)}^{2} \leq \mathfrak{s}_{N_{0}}(h)\|v\|_{L^{2}(\Omega)}^{2} .
$$

Applying Corollary 4.10, we obtain for all $v \in \mathfrak{E}_{N_{0}}(h)$ :

$$
h\left\langle\left(D_{y}^{2}+\frac{\mu^{\prime \prime}\left(\zeta_{0}\right)}{2} y^{2}\right) \Pi_{0} v, \Pi_{0} v\right\rangle-C h^{9 / 8}\left\|\Pi_{0} v\right\|_{L^{2}(\Omega)}^{2} \leq \mathfrak{s}_{N_{0}}(h)\left\|\Pi_{0} v\right\|_{L^{2}(\Omega)}^{2} .
$$

With Corollary 4.11 and the min-max principle, we infer that:

$$
h\left(2 N_{0}-1\right) \sqrt{\frac{\mu^{\prime \prime}\left(\zeta_{0}\right)}{2}}-C h^{9 / 8} \leq \mathfrak{s}_{N_{0}}(h)
$$

and thus $k(n)=n$ in Proposition 4.3. This ends the proof of Theorem 4.1.

\subsection{Eigenvector asymptotics}

From Theorem 4.1, we obtain that the gap between the eigenvalues is of order $h$. Thus, combining the construction of Section 4.1 with the spectral theorem (see [24, Lemmas 1213, Section 9]), we can deduce approximation results for the eigenfunctions $\mathfrak{u}_{n}(h)$ of $\mathfrak{L}_{h}$. Let us denote by $\tilde{\mathfrak{u}}_{n}^{b}(h)$ and $\tilde{\mathfrak{u}}_{n}^{\sharp}(h)$ the $n$-th quasimode with one and two terms, respectively, constructed in Section 4.1: We recall

$$
\begin{aligned}
& \tilde{\mathfrak{u}}_{n}^{b}(h)(y, z)=f_{n}(y) v_{\zeta_{0}}(z), \\
& \tilde{\mathfrak{u}}_{n}^{\sharp}(h)(y, z)=f_{n}(y) v_{\zeta_{0}}(z)+\left.h^{1 / 2} y f_{n}(y) \partial_{\zeta} v_{\zeta}\right|_{\zeta=\zeta_{0}}(z) .
\end{aligned}
$$


Here $f_{n}$ is the $n$-th eigenfunction of the harmonic oscillator $H_{\text {harm }}$ cf. (4.16). It is straightforward that we can take

$$
f_{n}(y)=\psi_{n-1}\left(\left[\frac{\mu^{\prime \prime}\left(\zeta_{0}\right)}{2}\right]^{1 / 4} y\right), \quad n \geq 1
$$

with $\psi_{m}$ the Hermite function of rank $m$. Then there exists an eigenmode $\mathfrak{u}_{n}(h)$ such that

$$
\begin{aligned}
\left\|\mathfrak{u}_{n}(h)-\tilde{\mathfrak{u}}_{n}^{b}(h)\right\|_{L^{2}(\Omega)} & \leq C h^{1 / 2}\left\|\mathbf{u}_{n}(h)\right\|_{L^{2}(\Omega)}, \\
\text { and } \quad\left\|\mathfrak{u}_{n}(h)-\tilde{\mathfrak{u}}_{n}^{\sharp}(h)\right\|_{L^{2}(\Omega)} & \leq C h\left\|\mathbf{u}_{n}(h)\right\|_{L^{2}(\Omega)} .
\end{aligned}
$$

Setting

$$
\tilde{u}_{n, \theta}^{b}(s, t)=\tilde{\mathfrak{u}}_{n}^{b}(h)(y, z) \quad \text { and } \quad \tilde{u}_{n, \theta}^{\sharp}(s, t)=\tilde{\mathfrak{u}}_{n}^{\sharp}(h)(y, z),
$$

with $h=\tan \theta$ and $(s, t)$ given by the change of variables (3.3), we obtain quasimodes for $\mathcal{L}_{\theta}$ which satisfy, for suitable eigenvectors $u_{n, \theta}$ of $\mathcal{L}_{\theta}$

$$
\left\|u_{n, \theta}-\tilde{u}_{n, \theta}^{b}\right\|_{L^{2}(\Omega)} \leq C \theta^{1 / 2}\left\|u_{n, \theta}\right\|_{L^{2}(\Omega)} \quad \text { and } \quad\left\|u_{n, \theta}-\tilde{u}_{n, \theta}^{\sharp}\right\|_{L^{2}(\Omega)} \leq C \theta\left\|u_{n, \theta}\right\|_{L^{2}(\Omega)} .
$$

Remark 4.12 For the same reason (cf. [24]), we get an approximation at any order in power of $\theta^{1 / 2}$ of $u_{n, \theta}$ when $\theta$ goes to 0 (and which is given by the asymptotic series defining the quasimode).

\section{Finite element computation of eigenpairs}

In this section, we show computations of the eigenvalues $\sigma_{n}(\theta)$ which display numerically the asymptotics given by Theorem 1.7. We also illustrate the results given by Theorems 1.3, 1.4 on isotropic and anisotropic decay of the eigenvectors of $\mathcal{L}_{\theta}$.

The simulations have been realized with the Finite Element Library MÉLINA, see [16].

\subsection{Eigenvalues}

We illustrate here the behavior of the eigenvalues of $\mathcal{L}_{\theta}$. The operator $\mathcal{L}_{\theta}$ is defined on the infinite domain $\Omega=\mathbb{R} \times \mathbb{R}_{+}$. We bound this infinite domain by a large box $R_{a, b, c}:=$ $(-a, b) \times(0, c)$ to perform numerical approximations. We compute the eigenvalues denoted by $\sigma_{n}(\theta ; a, b, c)$ of the operator $\mathcal{L}_{\theta}(a, b, c)=-\Delta+V_{\theta}$ on $R_{a, b, c}$ with Neumann condition on $t=0$ and Dirichlet conditions on the artificial boundary $\{s=-a\} \cup\{s=b\} \cup\{t=c\}$. Using the inclusion of the form domain of $\mathcal{L}_{\theta}(a, b, c)$ in that of $\mathcal{L}_{\theta}$, we prove

$$
\sigma_{n}(\theta) \leq \sigma_{n}(\theta ; a, b, c) .
$$

Furthermore, by similar arguments, we obtain the monotonicity of $\sigma_{n}(\theta ; a, b, c)$ according to each variable $a, b$ or $c$. The method consists in computing for several sets of values of 
$(a, b, c)$ with several combinations of rectangular finite elements of different degrees until convergence is found.

Figure 1 gives an approximation of the first 4 eigenvalues of $\mathcal{L}_{\theta}$ below 1 . For this, the final choice of $(a, b, c)$ is $(100,100,100)$ with rectangular elements of degree $\mathbb{Q}_{10}$ and 15 elements in each direction.

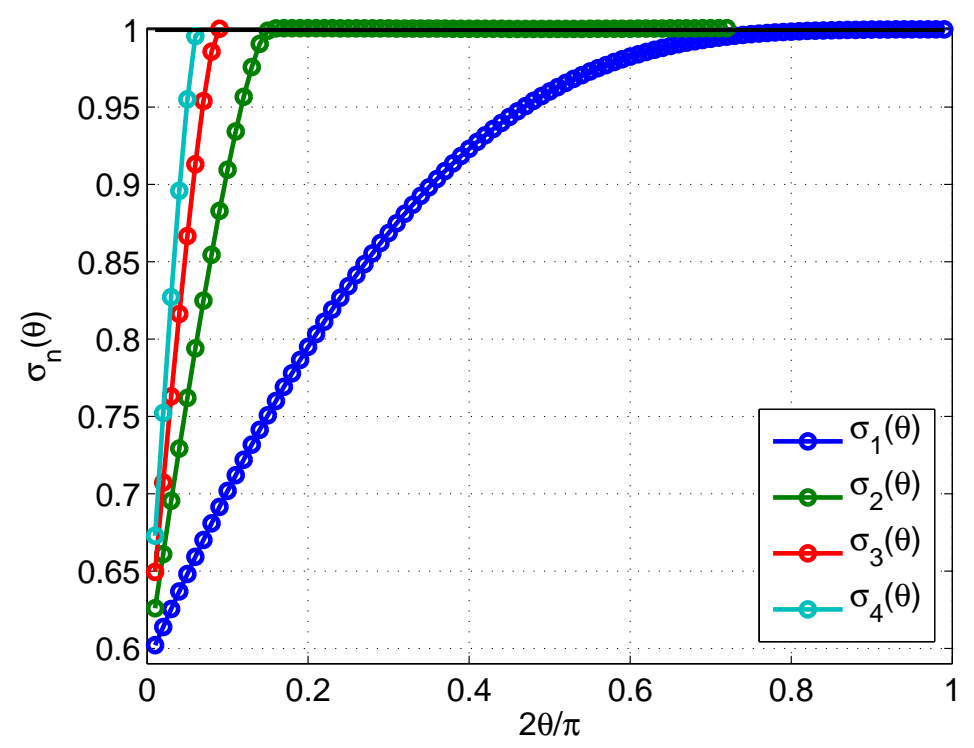

Figure 1: $\sigma_{n}(\theta ; 100,100,100)$ for $n=1, \ldots, 4$ (ordinates) versus $\vartheta=2 \theta / \pi$ (abscissa). Sampling: $\vartheta=k / 100,1 \leq k \leq 99$.

By looking at Figure 1, we can conjecture that the derivative of $\sigma_{1}(\theta)$ tends to zero when $\theta$ goes to $\frac{\pi}{2}$. This is true indeed, as we prove in the following proposition:

Proposition 5.1 For all $\theta \in\left(0, \frac{\pi}{2}\right)$, we have:

$$
\sigma_{1}(\theta) \cos \theta-\sigma_{1}^{\prime}(\theta) \sin \theta>0 .
$$

Moreover, we have:

$$
\lim _{\substack{\theta \rightarrow \frac{\pi}{2} \\ \theta<\frac{\pi}{2}}} \sigma_{1}^{\prime}(\theta)=0 .
$$

Proof: For $\gamma \geq 0$, we introduce the operator:

$$
\mathcal{L}(\theta, \gamma)=D_{s}^{2}+D_{t}^{2}+(t(\cos \theta+\gamma)-s \sin \theta)^{2}
$$

and we denote by $\sigma_{1}(\theta, \gamma)$ the bottom of its spectrum. Let $\rho>0$ and $\alpha \in\left(0, \frac{\pi}{2}\right)$ satisfy

$$
\cos \theta+\gamma=\rho \cos \alpha \quad \text { and } \quad \sin \theta=\rho \sin \alpha .
$$


We perform the rescaling $t=\rho^{-1 / 2} \hat{t}, s=\rho^{-1 / 2} \hat{s}$ and obtain that $\mathcal{L}(\theta, \gamma)$ is unitarily equivalent to:

$$
\rho\left(D_{\hat{s}}^{2}+D_{\hat{t}}^{2}+(\hat{t} \cos \alpha-\hat{s} \sin \alpha)^{2}\right)=\rho \mathcal{L}_{\alpha} .
$$

In particular, we observe that $\sigma_{1}(\theta, \gamma)=\rho \sigma_{1}(\alpha)$ is a simple eigenvalue: there holds

$$
\sigma_{1}(\theta, \gamma)=\sqrt{(\cos \theta+\gamma)^{2}+\sin ^{2} \theta} \sigma_{1}\left(\arctan \left(\frac{\sin \theta}{\cos \theta+\gamma}\right)\right) .
$$

Performing the rescaling $\tilde{t}=(\cos \theta+\gamma) t$, we get the operator $\tilde{\mathcal{L}}(\theta, \gamma)$ which is unitarily equivalent to $\mathcal{L}(\theta, \gamma)$ :

$$
\tilde{\mathcal{L}}(\theta, \gamma)=D_{s}^{2}+(\cos \theta+\gamma)^{2} D_{\tilde{t}}^{2}+(\tilde{t}-s \sin \theta)^{2} .
$$

We observe that the domain of $\tilde{\mathcal{L}}(\theta, \gamma)$ does not depend on $\gamma \geq 0$. Denoting by $\tilde{u}_{\theta, \gamma}$ the $L^{2}$-normalized and positive eigenfunction of $\tilde{\mathcal{L}}(\theta, \gamma)$ associated with $\sigma_{1}(\theta, \gamma)$, we write:

$$
\tilde{\mathcal{L}}(\theta, \gamma) \tilde{u}_{\theta, \gamma}=\sigma_{1}(\theta, \gamma) \tilde{u}_{\theta, \gamma}
$$

Taking the derivative with respect to $\gamma$, multiplying by $\tilde{u}_{\theta, \gamma}$ and integrating, we get the Feynman-Hellman formula:

$$
\partial_{\gamma} \sigma_{1}(\theta, \gamma)=2(\cos \theta+\gamma) \int_{\Omega}\left|D_{t} \tilde{u}_{\theta, \gamma}\right|^{2} d s d t \geq 0
$$

We deduce that, if $\partial_{\gamma} \sigma_{1}(\theta, \gamma)=0$, then $D_{t} \tilde{u}_{\theta, \gamma}=0$ and $\tilde{u}_{\theta, \gamma}$ only depends on $s$, which is a contradiction with $\tilde{u}_{\theta, \gamma} \in L^{2}(\Omega)$. Consequently, we have $\partial_{\gamma} \sigma_{1}(\theta, \gamma)>0$ for any $\gamma \geq 0$. An easy computation using formula (5.1) provides:

$$
\partial_{\gamma} \sigma_{1}(\theta, 0)=\sigma_{1}(\theta) \cos \theta-\sigma_{1}^{\prime}(\theta) \sin \theta
$$

As recalled in Subsection 1.1, the function $\sigma_{1}$ is analytic and increasing. Thus we deduce:

$$
\forall \theta \in\left(0, \frac{\pi}{2}\right), \quad 0 \leq \sigma_{1}^{\prime}(\theta)<\frac{\cos \theta}{\sin \theta} \sigma_{1}(\theta)
$$

We get:

$$
0 \leq \liminf _{\substack{\theta \rightarrow \frac{\pi}{2} \\ \theta<\frac{\pi}{2}}} \sigma_{1}^{\prime}(\theta) \leq \limsup _{\substack{\theta \rightarrow \frac{\pi}{2} \\ \theta<\frac{\pi}{2}}} \sigma_{1}^{\prime}(\theta) \leq 0
$$

which ends the proof. 




Figure 2: $\sigma_{n}(\theta ; 100,100,50)$ for $n=1, \ldots, 17$ (ordinates) versus $\vartheta=2 \theta / \pi$ (abscissa). Sampling: $\vartheta=k / 200,2 \leq k \leq 20$.

Figure 2 gives an approximation of all eigenvalues of $\mathcal{L}_{\theta}$ for small $\theta$. For this, the final choice of $(a, b, c)$ is $(100,100,50)$ with rectangular elements of degree $\mathbb{Q}_{10}$ and 20 elements in each direction. The figure corroborates the densification of the spectrum in $\left[\Theta_{0}, 1\right]$ described in Section 3.

We now illustrate formula (1.8). According to this formula, we have the convergence:

$$
\frac{\sigma_{n}(\theta)-\Theta_{0}}{a_{1} \theta} \rightarrow 2 n-1 \quad \text { as } \quad \theta \rightarrow 0, \quad \text { with } \quad a_{1}=\sqrt{\frac{\mu^{\prime \prime}\left(\zeta_{0}\right)}{2}},
$$

for all $n \geq 1$. Using numerical computations for $H_{\zeta}$, we find good approximations of $\Theta_{0}$ and $a_{1}$, cf. Tables 1-2 in [4]:

$$
\breve{\Theta}_{0}=0.590106125 \text { and } \breve{a}_{1} \simeq 0.7651881 .
$$

Let us denote by $\breve{\sigma}_{n}(\theta)$ the $n$-th computed eigenvalue of $\mathcal{L}_{\theta}$, with a convenient choice of the computational domain $(-a, b) \times(0, c)$. On Figure 3 we represent the functions

$$
\log 10(2 \theta / \pi) \longmapsto \rho_{n, 1}(\theta):=\frac{\breve{\sigma}_{n}(\theta)-\breve{\Theta}_{0}}{\breve{a}_{1} \theta}
$$

We can see that the ratio $\rho_{n, 1}(\theta)$ converges to $2 n-1$, corroborating formula (5.2).

Computations displayed in Figures 4 and 5 allow to evaluate the next terms of the asymptotic expansion for $\sigma_{n}(\theta)$. Indeed on Figure 4 we represent the functions

$$
\log 10(2 \theta / \pi) \longmapsto \rho_{n, 2}(\theta):=\frac{\breve{\Theta}_{0}+\breve{a}_{1}(2 n-1) \theta-\breve{\sigma}_{n}(\theta)}{\theta^{2}} .
$$




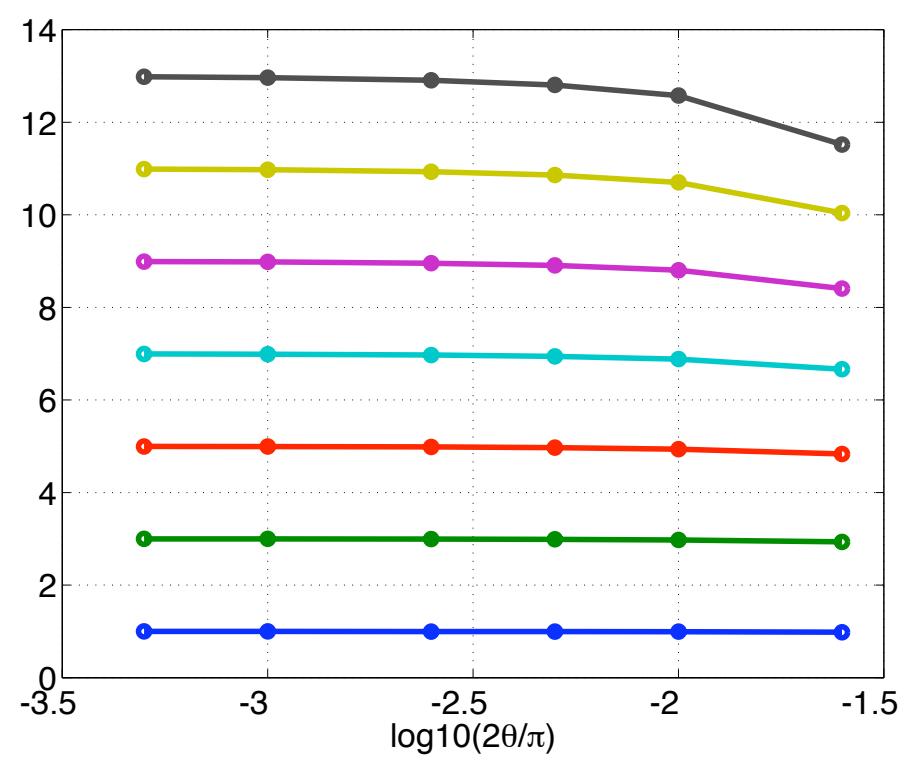

Figure 3: Convergence of $\rho_{n, 1}(\theta)$ to $2 n-1$ as $\theta \rightarrow 0, n=1, \ldots, 7$ (bottom to top).

We observe that the ratio $\rho_{n, 2}(\theta)$ converges to a numerical limit $\breve{a}_{n, 2}$ as $\theta \rightarrow 0$ for any $n=1, \ldots, 7$, see Table 1 .

\begin{tabular}{|c|ccccccc|}
\hline$n$ & 1 & 2 & 3 & 4 & 5 & 6 & 7 \\
\hline$\breve{a}_{n, 2}$ & 0.32616 & 1.1577 & 2.8206 & 5.3148 & 8.6402 & 12.797 & 17.784 \\
\hline
\end{tabular}

Table 1: Numerical limits $\breve{a}_{n, 2}$.

We can still determine numerically the next term of the expansion. On Figure 5 we represent the functions

$$
\log 10(2 \theta / \pi) \longmapsto \rho_{n, 3}(\theta):=\frac{\breve{\Theta}_{0}+\breve{a}_{1}(2 n-1) \theta-\breve{a}_{n, 2} \theta^{2}-\breve{\sigma}_{n}(\theta)}{\theta^{3}}
$$

Figure 5 agrees with the first terms of the asymptotics proved in Theorem 1.7 :

$$
\sigma_{n}(\theta)=\Theta_{0}+a_{1}(2 n-1) \theta-a_{n, 2} \theta^{2}-a_{n, 3} \theta^{3}+o\left(\theta^{3}\right)
$$




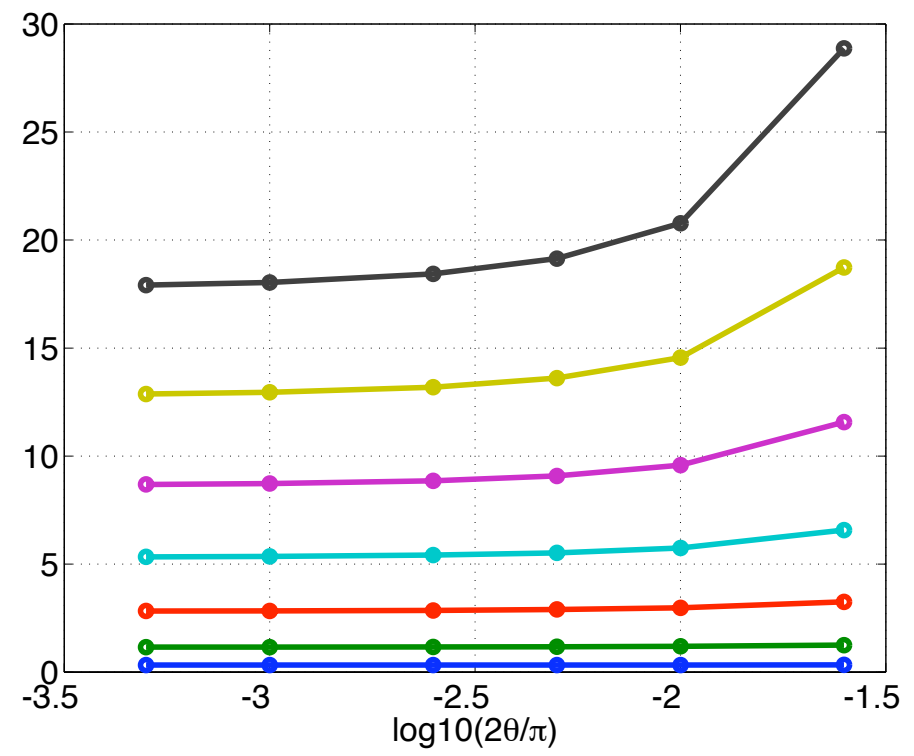

Figure 4: $\rho_{n, 2}(\theta)$ (ordinates) versus $\log 10(2 \theta / \pi)$ (abscissa). $n=1, \ldots, 7$ (bottom to top).

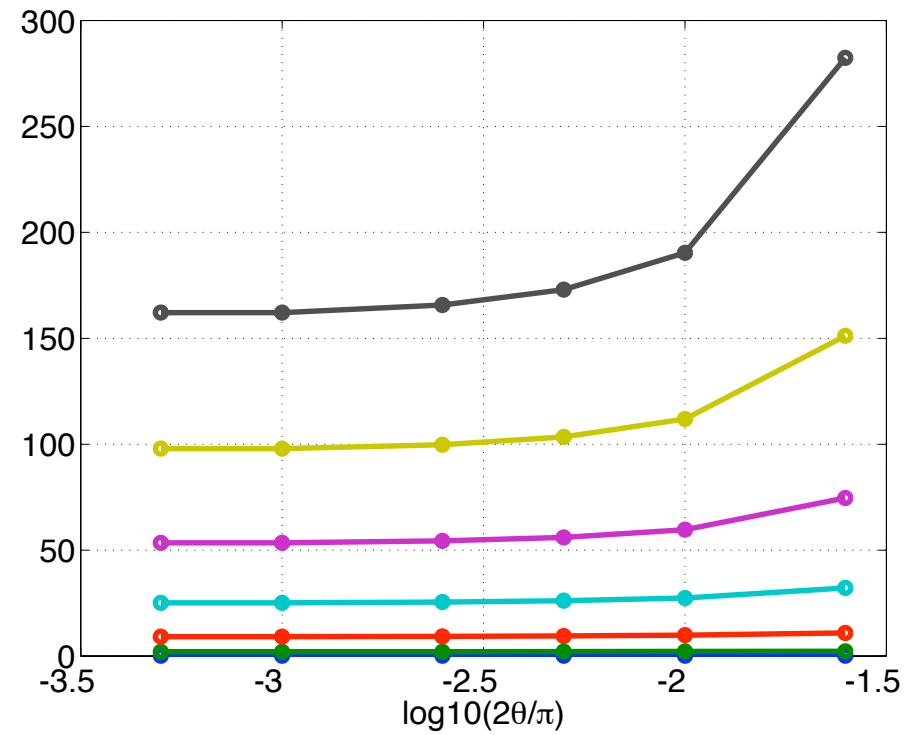

Figure 5: $\rho_{n, 3}(\theta)$ (ordinates) versus $\log 10(2 \theta / \pi)$ (abscissa). $n=1, \ldots, 7$ (bottom to top). 


\subsection{Eigenvectors}

In this section, we highlight the isotropic exponential decay of the eigenvectors of $\mathcal{L}_{\theta}$ and the anisotropic one given respectively in Theorems 1.3 and 1.4. Figure 6 illustrates the anisotropic decay for $\theta$ close to $\pi / 2:$ we compute the first eigenpair of $\mathcal{L}_{\theta}(5,15,75)$ on $[-5,15] \times[0,75]$ for $\theta=\vartheta \pi / 2, \vartheta=0.9,0.85,0.8,0.7$ with unit square elements of degree $\mathbb{Q}_{2}$. The first eigenvector is localized along the line $V_{\theta}=0$ and we see the exponential decay far away from this line. When $\theta$ is close to $\pi / 2$, the eigenvector spreads along the line $V_{\theta}=0$ and the exponential decay far away from the origin is not predominant.

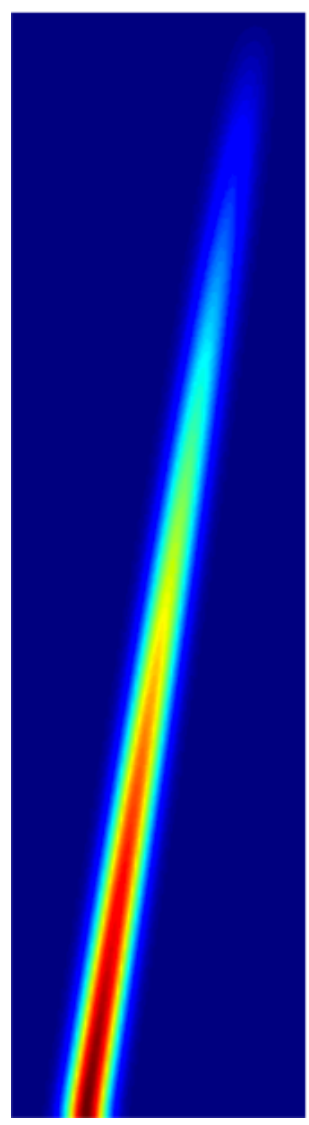

$\breve{\sigma}_{1}(\theta)$

1.0001656284

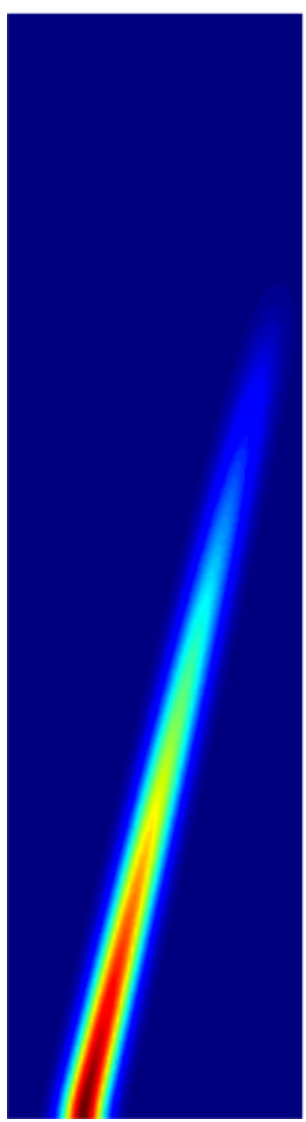

0.99987798948

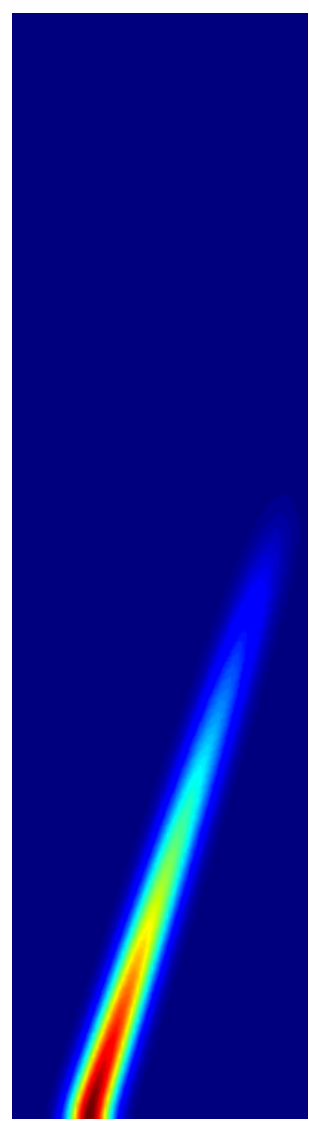

0.99910390126

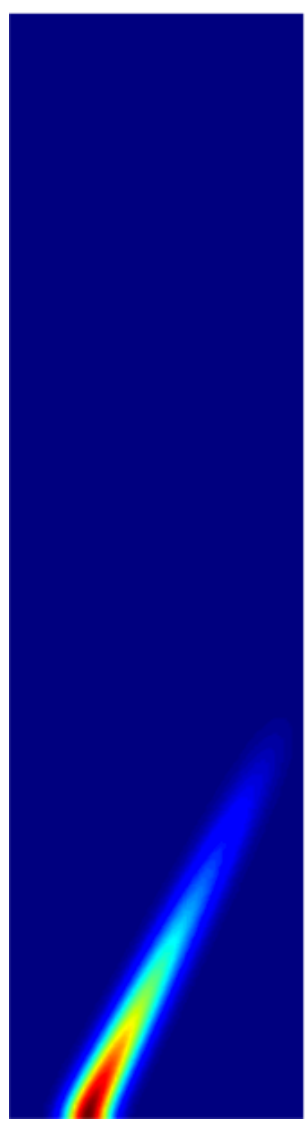

0.99445407220

Figure 6: First eigenmode of $\mathcal{L}_{\theta}$ for $\theta=\vartheta \pi / 2$ with $\vartheta=0.9,0.85,0.8$ and 0.7 .

When $\theta=\vartheta \pi / 2$ with $\vartheta \in\{0.1, \ldots, 0.6\}$, we observe equivalently the decay far away from the line $V_{\theta}=0$ and the origin (see (1.4) and (1.5)). Figure 7 gives an approximation of the first eigenvector and eigenvalue of $\mathcal{L}_{\theta}$ on the computational domain $[-15,25] \times[0,15]$ with unit square elements of degree $\mathbb{Q}_{6}$. We observe also that the first eigenvector spreads less and less along the line $V_{\theta}=0$ when $\theta$ is decreasing and the decay becomes essentially radial. 




0.98432278339

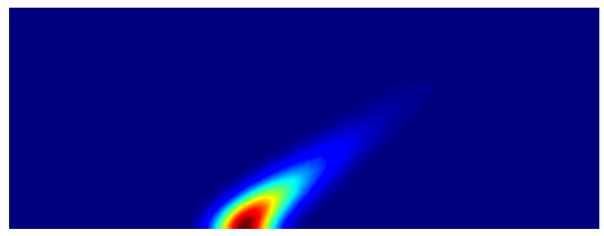

0.92410049174

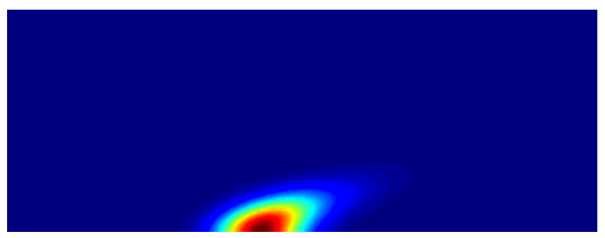

0.79630376085



0.96110511136

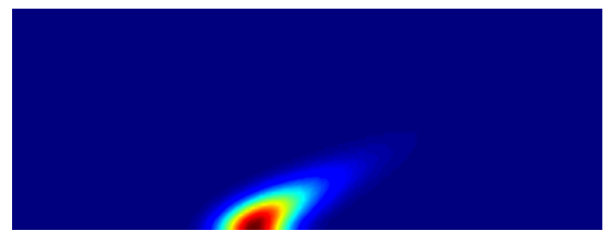

0.86980918147

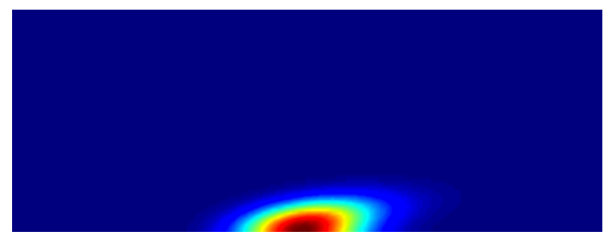

0.70307031204

Figure 7: First eigenmode of $\mathcal{L}_{\theta}$ for $\theta=\vartheta \pi / 2$ with $\vartheta=0.6,0.5,0.4,0.3,0.2$ and 0.1 .

On Figure 8, 9, and 10 we consider the asymptotics $\theta \rightarrow 0$. Figure 8 gives an approximation of the first eight eigenmodes of $\mathcal{L}_{\theta}$ computed by a finite element method with rectangular $2 \times 1$ elements and degree $\mathbb{Q}_{6}$ on the domain $[-20,80] \times[0,10]$. The oscillations with respect to the horizontal variable appear clearly. We can compare with Figures 9 and 10 where are represented the quasimodes ${ }^{1} \tilde{u}_{n, \theta}^{b}$ and $\tilde{u}_{n, \theta}^{\sharp}$ introduced in (4.38)-(4.42). We observe an interesting correlation between the computed eigenvectors on Figure 8 and the quasimodes on Figures 9 and 10.

\footnotetext{
${ }^{1}$ To compute these quasimodes, we approximate the one-dimensional eigenvector $v_{\zeta_{0}}$ with a finite difference method for the operator $D_{t}^{2}+\left(t-\zeta_{0}\right)^{2}$ on $[0,10]$ with Dirichlet condition on $t=10$, and $\zeta_{0}=0.76818365314$ according to computations of [4].
} 
0.619644

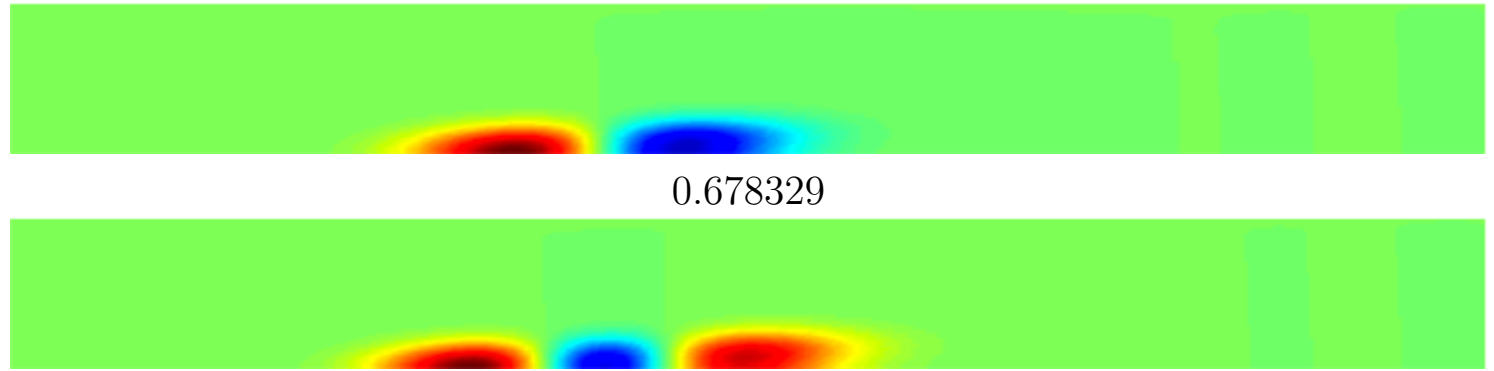

0.735340

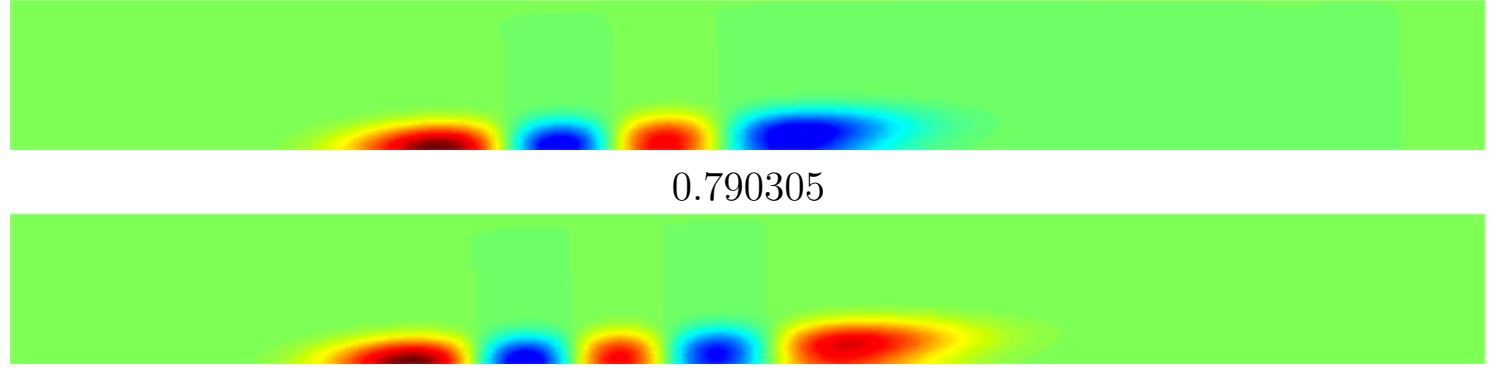

0.842702

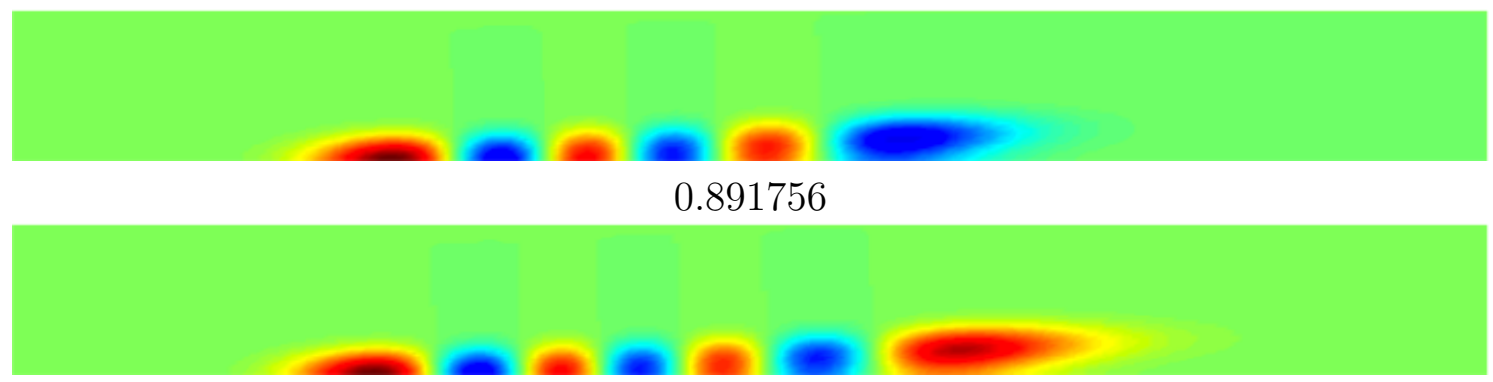

0.936217



0.973766

Figure 8: First 8 computed eigenvectors and eigenvalues of $\mathcal{L}_{\theta}$ for $\theta=0.0125 \pi$. 
0.620155

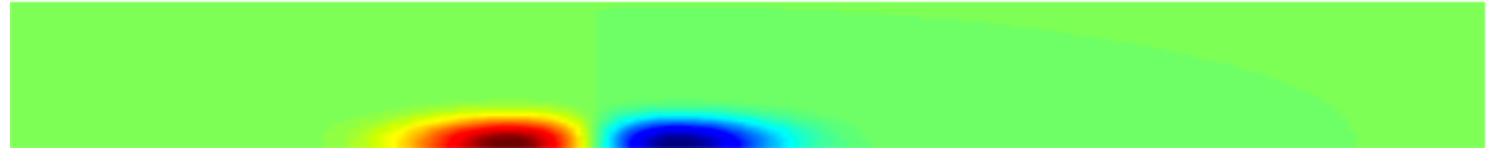

0.680253



0.740350

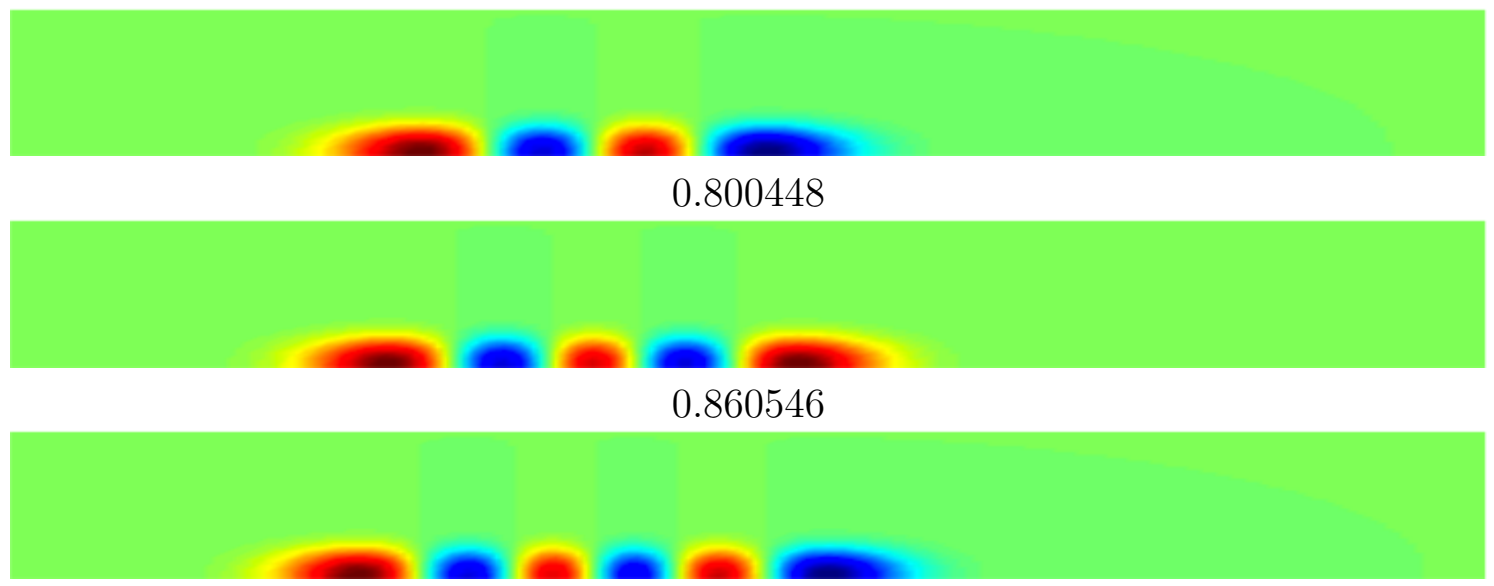

0.920644

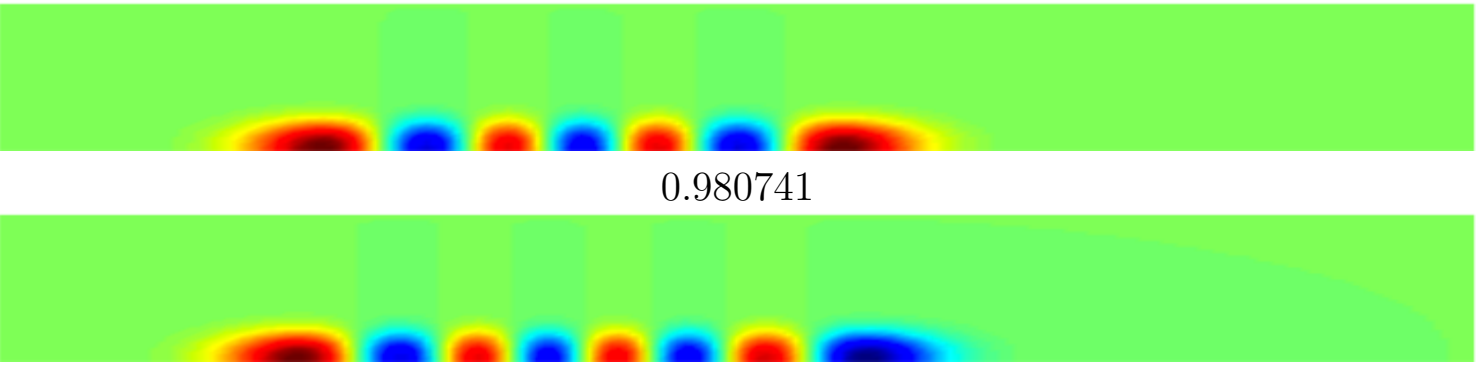

1.040839

Figure 9: Quasimodes $\tilde{u}_{n, \theta}^{b}$ and quasieigenvalues $\breve{\Theta}_{0}+\breve{a}_{1}(2 n-1) \theta$ for $\theta=0.0125 \pi$. 
0.620155

0.680253



0.740350

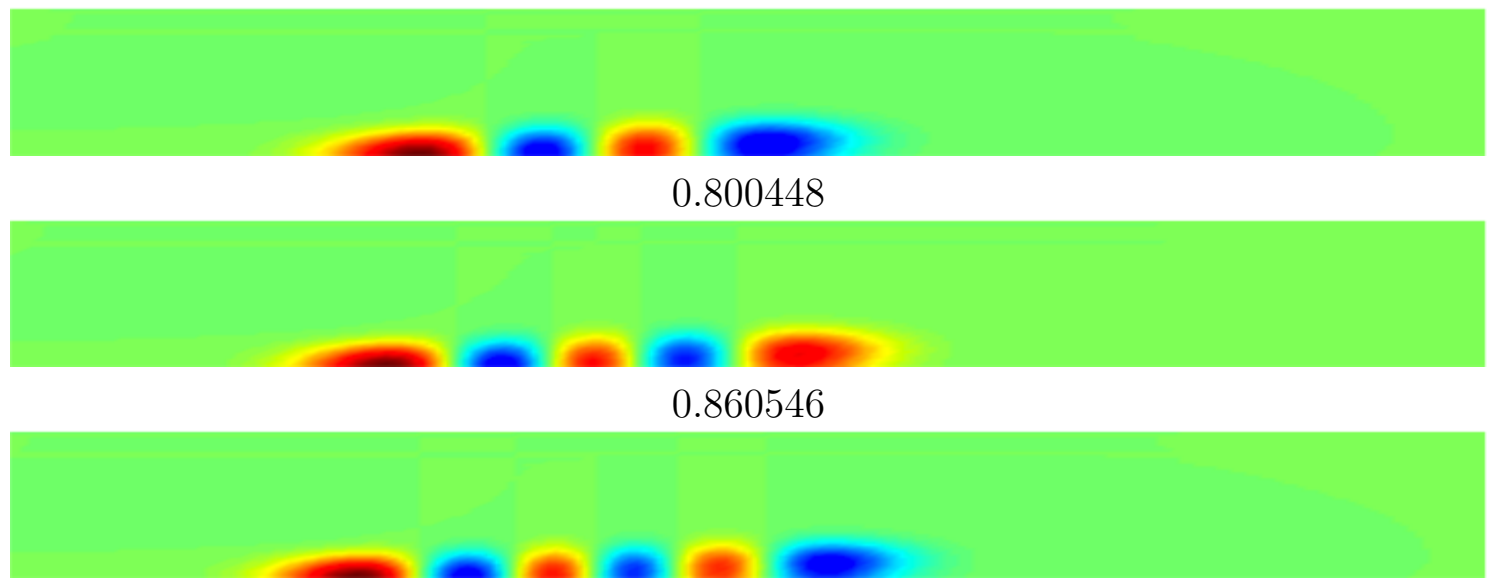

0.920644

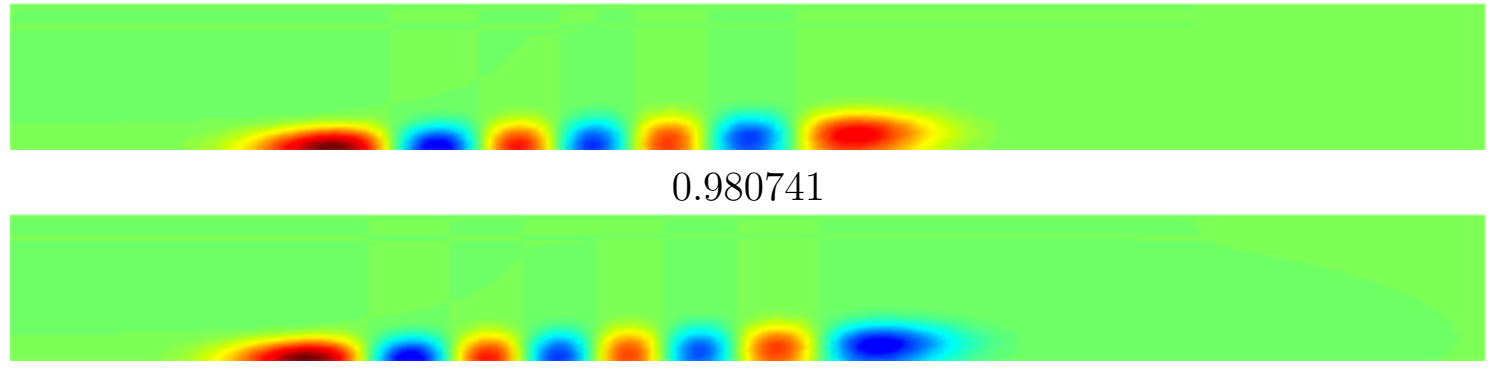

1.040839

Figure 10: Quasimodes $\tilde{u}_{n, \theta}^{\sharp}$ and quasieigenvalues $\breve{\Theta}_{0}+\breve{a}_{1}(2 n-1) \theta$ for $\theta=0.0125 \pi$. 


\section{References}

[1] S. Agmon. Lectures on exponential decay of solutions of second-order elliptic equations: bounds on eigenfunctions of $N$-body Schrödinger operators, volume 29 of Mathematical Notes. Princeton University Press, Princeton, NJ 1982.

[2] S. Agmon. Bounds on exponential decay of eigenfunctions of Schrödinger operators. In Schrödinger operators (Como, 1984), volume 1159 of Lecture Notes in Math., pages 1-38. Springer, Berlin 1985.

[3] A. Bernoff, P. Sternberg. Onset of superconductivity in decreasing fields for general domains. J. Math. Phys. 39(3) (1998) 1272-1284.

[4] V. Bonnaillie-Nö̈L. Harmonic oscillators with Neumann condition on the halfline. Commun. Pure Appl. Anal. To appear (2011).

[5] B. Chenaud, P. Duclos, P. Freitas, D. KrejčiŘíí. Geometrically induced discrete spectrum in curved tubes. Differential Geom. Appl. 23(2) (2005) 95-105.

[6] H. L. Cycon, R. G. Froese, W. Kirsch, B. Simon. Schrödinger operators with application to quantum mechanics and global geometry. Texts and Monographs in Physics. Springer-Verlag, Berlin, study edition 1987.

[7] M. Dauge, B. HelfFer. Eigenvalues variation. I. Neumann problem for SturmLiouville operators. J. Differential Equations 104(2) (1993) 243-262.

[8] P. Duclos, P. ExNER. Curvature-induced bound states in quantum waveguides in two and three dimensions. Rev. Math. Phys. 7(1) (1995) 73-102.

[9] S. Fournais, B. HelfFer. Spectral methods in surface superconductivity. Progress in Nonlinear Differential Equations and their Applications, 77. Birkhäuser Boston Inc., Boston, MA 2010.

[10] B. HELFFER. Semi-classical analysis for the Schrödinger operator and applications, volume 1336 of Lecture Notes in Mathematics. Springer-Verlag, Berlin 1988.

[11] B. Helffer, A. Morame. Magnetic bottles for the Neumann problem: the case of dimension 3. Proc. Indian Acad. Sci. Math. Sci. 112(1) (2002) 71-84. Spectral and inverse spectral theory (Goa, 2000).

[12] B. Helffer, J. SjÖstrand. Multiple wells in the semiclassical limit. I. Comm. Partial Differential Equations 9(4) (1984) 337-408.

[13] M. E. H. ISMAIL, R. ZHANG. On the Hellmann-Feynman theorem and the variation of zeros of certain special functions. Adv. in Appl. Math. 9(4) (1988) 439-446. 
[14] T. Kato. Perturbation theory for linear operators. Classics in Mathematics. Springer-Verlag, Berlin 1995. Reprint of the 1980 edition.

[15] K. Lu, X.-B. PAN. Surface nucleation of superconductivity in 3-dimensions. J. Differential Equations 168(2) (2000) 386-452. Special issue in celebration of Jack K. Hale's 70th birthday, Part 2 (Atlanta, GA/Lisbon, 1998).

[16] D. MARTIN. Mélina, bibliothèque de calculs éléments finis. http://anum-maths.univrennes1.fr/melina (2010).

[17] A. Martinez. Développements asymptotiques et effet tunnel dans l'approximation de Born-Oppenheimer. Ann. Inst. H. Poincaré Phys. Théor. 50(3) (1989) 239-257.

[18] A. Morame, F. Truc. Remarks on the spectrum of the Neumann problem with magnetic field in the half-space. J. Math. Phys. 46(1) (2005) 012105, 13.

[19] A. Persson. Bounds for the discrete part of the spectrum of a semi-bounded Schrödinger operator. Math. Scand. 8 (1960) 143-153.

[20] N. RAYMOND. Sharp asymptotics for the Neumann Laplacian with variable magnetic field: case of dimension 2. Ann. Henri Poincaré 10(1) (2009) 95-122.

[21] N. RAYMOND. On the semi-classical 3D Neumann Laplacian with variable magnetic field. Asymptotic Analysis 68(1-2) (2010) 1- 40.

[22] M. ReED, B. Simon. Methods of modern mathematical physics. IV. Analysis of operators. Academic Press [Harcourt Brace Jovanovich Publishers], New York 1978.

[23] M. Schatzman. Analyse numérique. InterEditions, Paris 1991. Cours et exercices pour la licence. [Course and exercises for the bachelor's degree].

[24] M. I. VIŠIK, L. A. LJUSTERNIK. Regular degeneration and boundary layer for linear differential equations with small parameter. Amer. Math. Soc. Transl. (2) 20 (1962) 239-364. 